\title{
Residual-augmented IVX predictive regression
}

\section{Working Papers 2016}

BANCO DE PORTUGAL Matei Demetrescu|Paulo M. M. Rodrigues

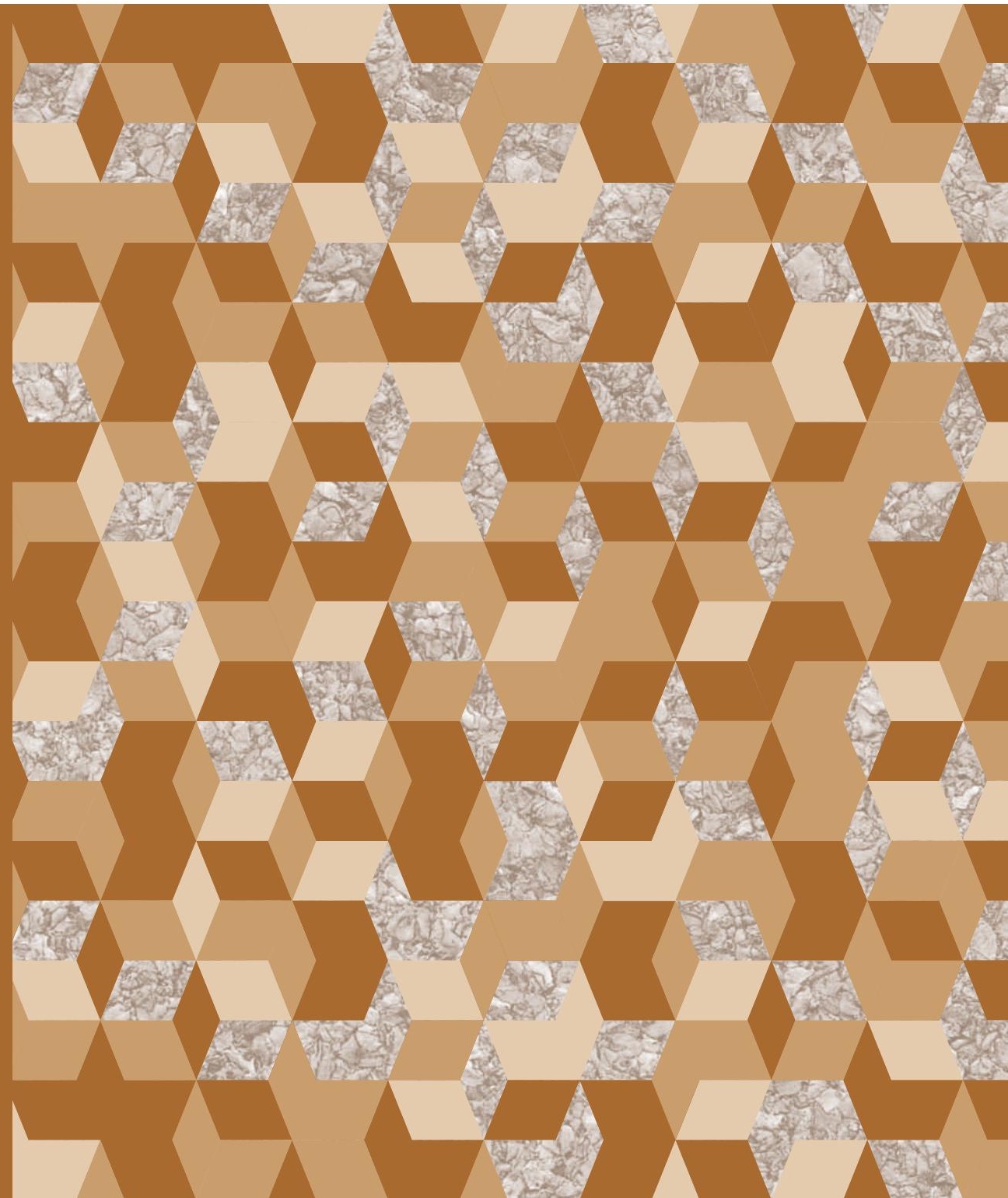





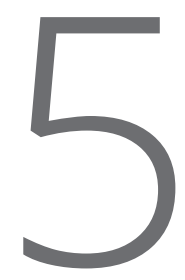

\section{Residual-augmented IVX predictive regression}

\section{Working Papers 2016}

Matei Demetrescu | Paulo M. M. Rodrigues

The analyses, opinions and findings of these papers represent the views of the authors, they are not necessarily those of the Banco de Portugal or the Eurosystem

Please address correspondence to

Banco de Portugal, Economics and Research Department

Av. Almirante Reis 71, 1150-012 Lisboa, Portugal

T+351213130000 | estudos@bportugal.pt

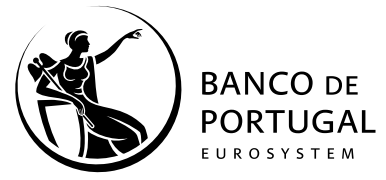

Lisbon, 2016 • www.bportugal.pt 
WORKING PAPERS | Lisbon 2016 • Banco de Portugal Av. Almirante Reis, 71 | 1150-012 Lisboa • www.bportugal.pt • Edition Economics and Research Department • ISBN 978-989-678-413-3 (online) • ISSN 2182-0422 (online) 


\title{
Residual-augmented IVX predictive regression
}

\author{
Matei Demetrescu \\ Christian-Albrechts-University of Kiel
}

\author{
Paulo M. M. Rodrigues \\ Banco de Portugal \\ NOVA School of Business and \\ Economics
}

January 2016

\begin{abstract}
Bias correction in predictive regressions stabilizes the empirical size properties of OLSbased predictability tests. This paper shows that bias correction also improves the finite sample power of tests, in particular so in the context of the extended instrumental variable (IVX) predictability testing framework introduced by Kostakis et al. (2015, Review of Financial Studies). We introduce new IVX-statistics subject to a bias correction analogous to that proposed by Amihud and Hurvich (2014, Journal of Financial and Quantitative Analysis). Three important contributions are provided: first, we characterize the effects that bias-reduction adjustments have on the asymptotic distributions of the IVX test statistics in a general context allowing for short-run dynamics and heterogeneity; second, we discuss the validity of the procedure when predictors are stationary as well as nearintegrated; and third, we conduct an exhaustive Monte Carlo analysis to investigate the small-sample properties of the test procedure and its sensitivity to distinctive features that characterize predictive regressions in practice, such as strong persistence, endogeneity, non-Gaussian innovations and heterogeneity. An application of the new procedure to the Welch and Goyal (2008) database illustrates its usefulness in practice.
\end{abstract}

JEL: C12, C22

Keywords: Predictability, persistence, persistence change, bias reduction.

E-mail: mdeme@stat-econ.uni-kiel.de; pmrodrigues@bportugal.pt 


\section{Introduction}

Predictive regressions are widely used in economics and finance; see, e.g., Campbell (2008) and Phillips (2015) for surveys. Typically, the variable of interest is regressed on lagged values of a predictor and the existence of predictability assessed through the statistical significance of the resultant estimate of the corresponding slope parameter. However, two important features of predictors need to be taken into consideration in this analysis: i) many predictors are often characterized by highly persistent autoregressive dynamics, and ii) many predictors also exhibit innovations which are strongly correlated to the innovations of the dependent variable. These features raise serious problems of endogeneity which can lead to sizeably biased estimates in finite samples (Stambaugh 1986 and Mankiw and Shapiro 1986) and to substantial over-rejections of the null hypothesis of no predictability. The usual asymptotic approximation employing the (standard) normal distribution performs particularly bad when predictors are persistent, even though the largest autoregressive roots of the typical predictor candidate are usually smaller than one - reason for which near-integrated asymptotics has been favoured as an alternative framework for inference (Elliott and Stock 1994 and Campbell and Yogo 2006). In the context of near-integrated regressors, the limiting distribution of the slope parameter estimator is not centered at zero, and this bias depends on the mean reversion parameter of the nearintegrated regressor. Although near-integrated asymptotics approximates the finite-sample behavior of the $t$-statistic for no predictability considerably better when predictors are persistent, the exact degree of persistence of a given predictor, and thus the correct critical values for a predictability test, are not known in advance. Moreover, standard estimation or pretests also fail in this context (Cavanagh et al. 1995).

These difficulties have led to the proposal of a number of alternative approaches, which differ mainly in the assumptions that characterize the stochastic properties of predictors (i.e., whether these are stationary or nearintegrated); see for instance, Campbell and Yogo (2006); Jansson and Moreira (2006); Maynard and Shimotsu (2009); Camponovo (2015); Breitung and Demetrescu (2015) and references therein. The recently proposed extended instrumental variable estimation approach [denoted IVX] motivated by 
Magdalinos and Phillips (2009) is becoming increasingly popular in predictive regressions, especially because the relevant $t$-statistic exhibits the same limiting distribution in both, stationary and near-integrated setups and is in this sense invariant to persistence; see, e.g., Kostakis et al. (2015); Gonzalo and Pitarakis (2012); Lee (2012) and Phillips and Lee (2013). The reasoning behind the approach consists in the generation of an instrumental variable whose persistence can be controlled, and this is achieved by suitably filtering the actual predictor.

To some extent, all methods lose some power by having to robustify against unknown persistence; however, as illustrated by Kostakis et al. (2015) the IVX methodology offers a good balance between size control and power loss. Since the noise-to-signal ratio in predictive regressions is quite high, one should still strive to improve this balance. For instance, Demetrescu (2014b) uses a simple variable addition scheme to improve the convergence rates of IVX estimators (and thus the local power of the corresponding $t$-tests) when the instrument used is relatively close to stationarity. However, for instrument choices closer to near-integration a different approach is required to improve the finite sample power of IVX-based tests without giving up size control.

To this end, we take a closer look at the class of reduced-bias techniques proposed by Amihud and Hurvich (2004) and extended by Amihud et al. (2009, 2010); see, inter alia, Bali (2008), Chun (2009), Avramov et al. (2010) and Johannes et al. (2014) for recent empirical applications building on this approach. When compared to other available procedures, the distinctive characteristic of these techniques is that they estimate the predictive slope coefficient and its standard error in a suitably augmented predictive regression, so that the bias is reduced to a minimum. While this bias correction was intended to stabilize the size properties of OLS-based predictability tests, we argue that it may also contribute to improve power, in particular so for IVXbased testing.

This paper discusses the large-sample behavior of IVX-statistics subject to bias correction, i.e., the implementation of IVX in an augmented predictive regression context analogous to that of Amihud and Hurvich (2004), considering both stationary and near integrated predictors. Our main objectives are threefold: i) to characterize the effects that our bias-reduction adjustments have on the asymptotic distribution of the IVX-statistics in a general context; ii) to 
establish the validity of the procedure when predictors are stationary as well as near-integrated; and iii) to provide an exhaustive Monte Carlo analysis to investigate the small-sample properties of the test procedures under distinctive conditions that characterize predictive regressions in practice, such as strong persistence, endogeneity, non-Gaussian innovations and heterogeneity, and to contrast them to the properties of available procedures, such as Amihud and Hurvich (2004), Campbell and Yogo (2006) and the IVX approach proposed by Kostakis et al. (2015). Finally, we revisit the data set used in Welch and Goyal (2008) to illustrate the application of the procedure.

The remainder of the paper is organized as follows. Section 2 briefly describes the characteristic features of predictive regressions and the biasreduction technique proposed by Amihud and Hurvich (2004), and gives a brief preview of the advantages of the residual-augmented IVX. Section 3 presents the large-sample theory under empirically relevant assumptions, including for instance heterogeneity and time-varying unconditional variances. Section 4 discusses the finite sample performance of several procedures used to test for predictability. Section 5 presents the analysis of the Welch and Goyal data, and section 6 summarizes and concludes. A technical appendix collects the proofs of the main theoretical statements put forward in the paper.

\section{Predictive regression framework and tests}

\subsection{The simplest model}

To illustrate the issues with predictive regressions in general and the advantages of our approach in particular, we start by considering the single predictor theoretical model set up analyzed in Stambaugh (1999) and adopted, among many others, by Amihud and Hurvich (2004) and Campbell and Yogo (2006).

This setting characterizes the joint dynamics of a stochastic process, $\left\{y_{t}\right\}_{t=2}^{T}$, and its posited predictor, $\left\{x_{t}\right\}_{t=1}^{T-1}$, in a two-equation linear system as,

$$
\begin{aligned}
& y_{t}=\beta x_{t-1}+u_{t}, \quad t=2, \ldots, T \\
& x_{t}=\rho x_{t-1}+v_{t}
\end{aligned}
$$


where the innovations $\xi_{t}:=\left(u_{t}, v_{t}\right)^{\prime}$ in the two-equation system are typically serially independent Gaussian distributed with mean zero and covariance matrix $\Sigma$.

In this setting, predictability is formally analyzed by examining whether the null hypothesis, $\mathrm{H}_{0}: \beta=0$, is statistically rejected through a $t$-statistic on the OLS estimate $\hat{\beta}$ computed from (1). The usual alternative hypothesis is that $\beta>0$, focusing on one-sided tests, but two-sided tests $\beta \neq 0$, are also frequently used in the literature. We shall refer to the resultant least-squares statistic as $t_{\hat{\beta}}$ in the sequel. It is a well-documented fact that when the correlation, $\frac{\sigma_{u v}}{\sigma_{u} \sigma_{v}}$, between innovations is large and $\rho \simeq 1$, the distribution of $t_{\hat{\beta}}$ largely departs from the typical standard normal limit, posing therefore an interesting challenge on inference; see, e.g., Elliott and Stock (1994) and Stambaugh (1999).

Specifically, under these simple assumptions, weak convergence of the partial sum of $\xi_{t}$ holds, i.e., $\frac{1}{\sqrt{T}} \sum_{t=1}^{[s T]}\left(u_{t}, v_{t}\right)^{\prime} \Rightarrow\left(\sigma_{u} W_{u}(s), \sigma_{v} W_{v}(s)\right)^{\prime}$, where $\left(W_{u}(s), W_{v}(s)\right)^{\prime}$ is a vector of dependent standard Wiener processes (see, e.g., Davidson 1994, Chapter 29). Furthermore, considering that the autoregressive coefficient $\rho$ is local to unity, $\rho:=1-\frac{c}{T}$, we have, jointly with the above weak convergence, that $\frac{1}{\sqrt{T}} x_{[s T]} \Rightarrow B_{c}(s)$, where $B_{c}$ is an Ornstein-Uhlenbeck [OU] process driven by $W_{v}(s)$, i.e., $B_{c}(s):=W_{v}(s)-c \int_{0}^{s} e^{-c(s-r)} W_{v}(r) \mathrm{d} r$. Given these results it follows that the limiting distribution of the OLS based t-test, $t_{\hat{\beta}}$, computed from (1) when the predictor is near-integrated is given by

$$
t_{\hat{\beta}} \Rightarrow \sqrt{1-\frac{\sigma_{u v}^{2}}{\sigma_{u}^{2} \sigma_{v}^{2}}} \mathcal{Z}+\frac{\sigma_{u v}}{\sigma_{u} \sigma_{v}} \frac{\int_{0}^{1} B_{c}(s) \mathrm{d} W_{v}(s)}{\sqrt{\int_{0}^{1} B_{c}^{2}(\mathrm{~s}) \mathrm{d} s}}
$$

where $\mathcal{Z}$ is a standard normal variate independent of the Wiener process $W_{v}(r)$ driving $B_{c}(r)$.

REMARK 1. The assumptions of normality and serial independence allow for considerable simplification of the exposition, but shall be relaxed in the following section by allowing for more general forms of serial dependence or heterogeneity.

\subsection{Residual Augmented Predictive Regressions}

Considering (1) - (2) and stationarity of $\left\{x_{t}\right\}$, i.e., the additional assumption that $\rho$ in $(2)$ is fixed and satisfies $|\rho|<1$, Stambaugh $(1986,1999)$ shows that 
the exact OLS bias of $\hat{\beta}$ in 1 is $\gamma \mathrm{E}(\hat{\rho}-\rho)$, with $\hat{\rho}$ denoting the OLS estimate of $\rho$ and $\gamma:=\sigma_{u v} / \sigma_{v}^{2}$ is the slope coefficient in a regression of $u_{t}$ on $v_{t}$. Since $\hat{\rho}$ is known to be downward biased in small-samples, and $\left(u_{t}, v_{t}\right)^{\prime}$ are typically highly negatively contemporaneously correlated, the autoregressive OLS bias feeds into the small-sample distribution of $\hat{\beta}$ causing over-rejections of the null hypothesis of no predictability, $\mathrm{H}_{0}: \beta=0$.

To correct for this effect, Amihud and Hurvich (2004) propose a simple statistical device that builds upon the OLS estimates obtained from a predictive regression which is augmented with estimates of $v_{t}$, the innovations to the predictor in (2). The initial motivation for this type of augmentation is that the null distribution of the $t$-statistic on $\hat{\beta}$ in the infeasible regression

$$
y_{t}=\beta x_{t-1}+\gamma v_{t}+\varepsilon_{t}
$$

converges asymptotically to a standard normal distribution irrespectively of the stochastic nature of $x_{t}$ and the degree of contemporaneous correlation of $\left(u_{t}, v_{t}\right)^{\prime}$. Although it is tempting to use some proxy of $v_{t}$ to make this regression feasible, it should be noted that the appealing asymptotic properties of the infeasible test do not automatically extend to the feasible counterpart resulting from the use of the OLS residuals from (2), say $\hat{v}_{t}$. The reason is that the bias of $\hat{\rho}$ still feeds into the estimation of $\beta$ via $\hat{v}_{t}=v_{t}-(\hat{\rho}-\rho) x_{t-1}$ and, as a result, the distribution of the OLS $t$-statistic for $\beta=0$ in this regression, is simply a re-scaling of that of $t_{\hat{\beta}}$; see Rodrigues and Rubia (2011); Cai and Wang (2014) and Demetrescu (2014a), for further details.

The distinctive feature of the Amihud and Hurvich (2004) [AH] procedure is that it uses a bias-adjusted estimate of $v_{t}$ to reduce the bias of $\hat{\beta}$. Thus, the resulting feasible regression becomes,

$$
y_{t}=\beta x_{t-1}+\gamma \hat{v}_{t}^{*}+\varepsilon_{t},
$$

where $\hat{v}_{t}^{*}:=x_{t}-\hat{\rho}^{*} x_{t-1}$, with $\hat{\rho}^{*}$ denoting finite-sample bias-corrected OLS estimates of $\rho$ in (2). The central idea is to obtain a $\hat{\rho}^{*}$ as close to unbiasedness as possible. The procedure however also requires a correction in the form of specific standard errors which is not easily generalized to higher-order dynamics; see Amihud et al. $(2009,2010)$. 
REMARK 2. Augmenting linear regression models with covariates is often motivated in terms of efficiency gains (Faust and Wright 2011). Arguably, the primary purpose of the residual-augmented regression in (4) is to stabilize size, with power gains playing a secondary role. This is partly because the true process of the errors is unobservable and must be replaced by some empirical proxy (which prompts the correction for ensuring size control of the $\mathrm{AH}$ procedure). We argue in the following that power gains can indeed be expected in the IVX framework, while at the same time controlling for size.

\subsection{The IVX Test Procedures}

2.3.1. The Original IVX Approach. Our interest lies in the evaluation of the impact that the bias correction through augmentation may have on the IVX approach. The IVX procedure, introduced to predictive regressions by Kostakis et al. (2015), centers on the construction of instrumental variables from the potential predictors. This ensures relevance of the instruments while at the same time controlling for persistence. In particular, for the implementation of the procedure, one uses

$$
z_{t}:=(1-\varrho L)_{+}^{-1} \Delta x_{t}=\sum_{j=0}^{t-2} \varrho^{j} \Delta x_{t-j}
$$

as instrument for $x_{t}$, with $L$ standing for the conventional lag operator; the idea is to choose $\varrho:=1-\frac{a}{T^{\eta}}$, with $0<\eta \leq 1$, and $a \geq 0$ and fixed, such that $z_{t}$ is by construction only mildly integrated when the predictor $x_{t}$ is (nearly) integrated.

The resulting IVX estimator of $\beta$ (henceforth $\hat{\beta}^{i v x}$ ), computed from (1) using $z_{t}$ as instrument has a slower convergence rate than the conventional OLS estimator, but is mixed Gaussian in the limit irrespective of the degree of endogeneity implied by $\gamma$. This estimator is given by,

$$
\hat{\beta}^{i v x}:=\frac{\sum_{t=2}^{T} z_{t-1} y_{t}}{\sum_{t=2}^{T} z_{t-1} x_{t-1}}
$$

and its standard error is $s e\left(\hat{\beta}^{i v x}\right):=\frac{\hat{\sigma}_{u} \sqrt{\sum_{t=2}^{T} z_{t-1}^{2}}}{\sum_{t=2}^{T} z_{t-1} x_{t-1}}$; note that Kostakis et al. (2015) suggest the use of OLS residuals $\hat{u}_{t}$ (whose consistency properties do not depend on the persistence properties of the instrument $z_{t}$ ) for the computation of $\hat{\sigma}_{u}^{2}$. 
Breitung and Demetrescu (2015) analyse the power function of the IVXbased $t$-test, computed as $t_{i v x}:=\hat{\beta}^{i v x} / s e\left(\hat{\beta}^{i v x}\right)$, under local alternatives of the form $\beta:=\frac{b}{T^{1 / 2+\eta / 2}}$, and show that the limiting distribution under such local alternatives is

$$
t_{i v x} \Rightarrow \mathcal{Z}+b \frac{\sigma_{v} \sqrt{2}}{\sigma_{u} \sqrt{a}}\left[B_{c}^{2}(1)-\int_{0}^{1} B_{c}(s) \mathrm{d} B_{c}(s)\right]
$$

where $\mathcal{Z}$ is a standard normal variate independent of the OU process $B_{c}(r), a$ is the noncentrality parameter used in $\varrho$ for the construction of the instrument, and $\sigma_{v}$ and $\sigma_{u}$ are the standard deviations of $v_{t}$ and $u_{t}$, respectively. Note that the reduced convergence rate of $\hat{\beta}^{i v x}$ has consequences on the type of neighbourhoods where the IVX based test has nontrivial power. This, however, is the trade off for obtaining a pivotal limiting null distribution. While Kostakis et al. (2015) show that the power loss is moderate, one would of course prefer to reduce this loss as much as possible.

2.3.2. The Bias-reduced IVX Approach. Turning our attention to the bias correction approach proposed by Amihud and Hurvich (2004), note that, the residuals $\hat{v}_{t}^{*}$ used in the residual-augmented predictive regression in (4) rely on a bias-corrected estimate of $\rho$ in order to reduce the endogeneity of the predictor. Interestingly, since IVX uses a less persistent instrument for estimation than the original predictor, it turns out that in order to use the residual augmentation approach in the IVX framework it is not necessary to construct a bias corrected estimator, such as $\hat{\rho}^{*}$ used by Amihud and Hurvich (2004).This is an important advantage of the IVX procedure since it simplifies the analysis considerably and allows for easy generalisations to higher order dynamics in the predictor as we will show below.

REMARK 3. It may be surprising that, although simple augmentation using OLS residuals does not work for the OLS estimation of the predictive regression, it will work for IVX. Essentially, the estimation noise $\left(\hat{v}_{t}-v_{t}\right)$ does not affect the IVX estimator given the lower convergence rate of the latter compared to the OLS estimator. In fact, the improved local power is the same as if the true $v_{t}$ were used in (4): the local power of the test based on the augmented IVX regression is obtained by replacing $\sigma_{u}$ with $\sigma_{\varepsilon}$ in (6); see the next section for more details. Since $\sigma_{\varepsilon}<\sigma_{u}$ whenever $\gamma \neq 0$, we obtain by construction a 
larger drift term in the distribution under the local alternative $\beta:=b / T^{1 / 2+\eta / 2}$. This may not increase the convergence rate, but considering the typically high correlation of the innovations $u_{t}$ and $v_{t}$ (given by $\left.\frac{\sigma_{u v}}{\sigma_{u} \sigma_{v}}\right)$, the ratio $\left(\frac{\sigma_{u}}{\sigma_{\varepsilon}}\right)$ can be considerably larger than unity and power gains in finite samples are to be expected. This is confirmed in the Monte Carlo analysis in Section 4.

The implementation of our bias-reduced IVX approach in the simple introductory setup given by (1) and (2), is as follows:

1. Regress $x_{t}$ on $x_{t-1}$ to obtain the residuals $\hat{v}_{t}:=v_{t}-(\hat{\rho}-\rho) x_{t-1}$, where $\hat{\rho}:=\rho+\frac{\sum_{t=2}^{T} x_{t-1} v_{t}}{\sum_{t=2}^{T} x_{t-1}^{2}}$ is the usual OLS estimator.

2. Regress $y_{t}$ on $\hat{v}_{t}$ to obtain $\tilde{y}_{t}:=y_{t}-\hat{\gamma} \hat{v}_{t}=\varepsilon_{t}+\beta x_{t-1}+\gamma v_{t}-\hat{\gamma} \hat{v}_{t}$, where $\hat{\gamma}:=\frac{\sum_{t=2}^{T} \hat{v}_{t} y_{t}}{\sum_{t=2}^{T} \hat{v}_{t}^{2}}$ is the usual OLS estimator.

3. Regress $\tilde{y}_{t}$ on $x_{t-1}$ via IVX to obtain $\tilde{\beta}^{i v x}$ and the corresponding $t$-statistic, $\tilde{t}_{i v x}$; similarly to the original IVX, it helps if the residuals are computed using the OLS estimator, $\hat{\beta}$, of this regression given its consistency and higher convergence rates.

Remark 4. Considering $\tilde{y}_{t}$ as the dependent variable provides a convenient way to think about residual augmented predictive regressions. As discussed in Campbell and Yogo (2006), the unobservable process $\left[y_{t}-\mathrm{E}\left(u_{t} \mid v_{t}\right)\right]$ results from subtracting off the part of the innovation to the predictor variable that is correlated with $y_{t}$. This provides a less noisy dependent variable in the regression analysis and, therefore, yields power advantages over conventional predictive regressions that steam from a relative gain in statistical efficiency. In particular, since $\mathrm{E}\left(\varepsilon_{t}^{2}\right)=\left(1-\rho^{2}\right) \sigma_{u}^{2}$, the larger the degree of endogenous correlation in the system, the larger the amount of variability in the regressand not related to $x_{t-1}$ that can be filtered out - conversely, we can think of the standard predictive regression analysis as a particularly inefficient tool to detect predictability when $\rho$ is large. However, since $\left[y_{t}-\mathrm{E}\left(u_{t} \mid v_{t}\right)\right]$ cannot be directly observed, the feasible representation uses the OLS-based proxy $\tilde{y}_{t}$ in the equation.

REMARK 5. In practice, one may need to account for non-zero means of $y_{t}$; this is accomplished by including an intercept in the regression in step 2 and by demeaning the regressor $x_{t}$ in the IVX regression in step 3 (see Kostakis et al. 2015, for the justification of this demeaning procedure in step 3). In the 
near-integrated case, including an intercept in the autoregression in the first step is typically not needed for the kind of data one has in mind with stock return predictability, where deterministic trends are in general not an empirical issue.

Thus, following the three steps above we obtain the bias-corrected IVX estimator, viz.,

$$
\tilde{\beta}^{i v x}:=\frac{\sum_{t=2}^{T} z_{t-1} \tilde{y}_{t}}{\sum_{t=2}^{T} z_{t-1} x_{t-1}}=\hat{\beta}^{i v x}-\frac{\hat{\gamma} \sum_{t=2}^{T} z_{t-1} \widehat{v}_{t}}{\sum_{t=2}^{T} z_{t-1} x_{t-1}}
$$

and its corresponding standard error,

$$
\operatorname{se}\left(\tilde{\beta}^{i v x}\right):=q_{T} \frac{\hat{\sigma}_{\varepsilon} \sqrt{\sum_{t=2}^{T} z_{t-1}^{2}}}{\left|\sum_{t=2}^{T} z_{t-1} x_{t-1}\right|}
$$

where $\tilde{y}_{t}:=y_{t}-\widehat{\gamma} \widehat{v}_{t}, \hat{\sigma}_{\varepsilon}$ is the estimate of the standard deviation of $\varepsilon_{t}$ computed from the residuals $\tilde{\varepsilon}_{t}:=\tilde{y}_{t}-\hat{\beta} x_{t-1}$ and $\hat{\beta}:=\frac{\sum_{t=2}^{T} x_{t-1} \tilde{y}_{t}}{\sum_{t=2}^{T} x_{t-1}^{2}}$. Note that the estimator of the standard error in (8) includes the finite sample correction,

$$
q_{T}:=1+\frac{\left(\hat{\gamma} \hat{\sigma}_{v} \sum_{t=2}^{T} z_{t-1} x_{t-1}\right)^{2}}{\hat{\sigma}_{\varepsilon}^{2} \sum_{t=2}^{T} z_{t-1}^{2} \sum_{t=2}^{T} x_{t-1}^{2}} .
$$

A detailed discussion of the importance of $q_{T}$ will be presented in the following section, but it may be noted that (9) is in principle only required when the predictors used are stationary; see section 3 for details.

Hence, considering (7) and (8) inference can be performed based on the IVX $t$-statistic,

$$
\tilde{t}_{i v x}:=\tilde{\beta}^{i v x} / \operatorname{se}\left(\tilde{\beta}^{i v x}\right)
$$

which turns out to remain standard normal irrespectively of the stationarity or near-integratedness of the regressor.

\subsection{Short-run dynamics and heterogeneity}

This section looks into the properties of the residual-augmented IVX approach in the empirical relevant cases where predictors may display short-run dynamics and heterogeneity. Hence, in this section we lay out a fairly general setting, 
which is the framework we will use to characterise the asymptotic properties of the procedures introduced in this paper.

The starting question is how to deal with short-run dynamics in the increments of $x_{t}$, since this has implications as to which residuals to use for augmentation in the IVX testing procedure. Here, it is the innovations of $v_{t}$ (for which a finite-order AR process is a natural choice) that should correlate with $u_{t}$ rather than $v_{t}$ itself, like in the case without short-run dynamics. The augmentation approach (described in Section 2.2) relies on decomposing the shocks to the predictive regression as the sum of two orthogonal components; should $v_{t}$ be one of them, this induces serial correlation in $u_{t}$, which is not a plausible feature of the null hypothesis of no predictability. Hence, the general set up considered is formalized in the following assumptions.

Assumption 1. The data is generated according to (1) - (2) with initial condition $x_{1}$ bounded in probability.

Assumption 2. Let

$$
\left(\begin{array}{c}
\varepsilon_{t} \\
\nu_{t}
\end{array}\right):=\left(\begin{array}{c}
\sigma_{\varepsilon t} \xi_{\varepsilon t} \\
\sigma_{\nu t} \xi_{\nu t}
\end{array}\right)
$$

where $\left(\xi_{\varepsilon t}, \xi_{\nu t}\right)^{\prime}$ is a heterogeneous independent sequence with unity covariance matrix and, for some $\delta>0$, with uniformly bounded moments $\mathrm{E}\left(\left|\xi_{\varepsilon t}^{4+\delta}\right|\right)$ and $\mathrm{E}\left(\left|\xi_{\nu t}^{4+\delta}\right|\right)$. Furthermore, let $\sigma_{\varepsilon t}:=\sigma_{\varepsilon}\left(\frac{t}{T}\right)$ and $\sigma_{\nu t}:=\sigma_{\nu}\left(\frac{t}{T}\right)$, where $\sigma .(\cdot)$ are piecewise Lipschitz continuous functions, bounded away from zero.

Assumption 3. The errors $u_{t}$ and $v_{t}$ are given as

$$
\begin{aligned}
& v_{t}=a_{1} v_{t-1}+\ldots+a_{p-1} v_{t-p+1}+\nu_{t} \\
& u_{t}=\varepsilon_{t}+\gamma \nu_{t}, \quad t \in \mathbb{Z},
\end{aligned}
$$

where the innovations $\left(\varepsilon_{t}, \nu_{t}\right)^{\prime}$ are contemporaneously orthogonal white noise as indicated in Assumption 2.

Assumption 4. The autoregressive parameter $\rho$ is either i) fixed when $|\rho|<1$, or ii) time-varying near unity, $\rho:=1-\frac{c_{t}}{T}$ with $c_{t}:=c\left(\frac{t}{T}\right)$ and $c(\cdot)$ is a piecewise Lipschitz function. 
Assumption 2 acknowledges that time series (and in particular financial series) may exhibit permanent volatility changes, which is an important stylized fact of many financial series; see, among others, Guidolin and Timmermann (2006); Teräsvirta and Zhao (2011); Amado and Teräsvirta (2013) and Amado and Teräsvirta (2014). Such forms of nonstationarity typically invalidate the usual standard errors, ${ }^{1}$ and we resort to heteroscedasticity robust [HC] standard errors (also known as Eicker-White standard errors) to account for this feature. The use of White standard errors is also recommended by Kostakis et al. (2015) to deal with conditional heteroskedasticity - albeit under strict stationarity of the error series $v_{t}$. The $\operatorname{AR}(p-1)$ structure of $v_{t}$ in Assumption 3 is taken as an approximation to more general data generating processes [DGP]s. In theory, this would require letting $p \rightarrow \infty$ at suitable rates as $T \rightarrow \infty$; however, dealing with the asymptotics related to the order of augmentation determination is beyond the scope of this paper, but relevant results can be found, for instance, in Chang and Park (2002). Finally, Assumption 4 characterises the persistence properties of the predictor. The flexible near-integrated DGP resulting from Assumption 4 ii) is motivated by the high, yet uncertain persistence of typical predictor series. Moreover, since persistence is not always constant, in particular when close to the unit root region, we allow for time variation in persistence in the near integrated case.

Hence, the implementation of our residual-augmented IVX approach in the general framework described by Assumptions 1 through 4 consists of the following steps:

1. Compute the residuals $\hat{\nu}_{t}$ from an autoregressive model of order $p$ for the predictor $x_{t}$, viz.,

$$
\hat{\nu}_{t}=x_{t}-\sum_{j=1}^{p} \hat{\varphi}_{j} x_{t-j}=\nu_{t}-\sum_{j=1}^{p}\left(\hat{\varphi}_{j}-\varphi_{j}\right) x_{t-j}, \quad t=p+1, \ldots, T,
$$

with $\hat{\varphi}_{j}, j=1, \ldots, p$, the OLS autoregressive coefficient estimates. One may use some information criteria in levels to determine the autoregressive order $p$ (we use Akaike's information criteria (AIC) in sections 4 and 5); note that

1. This is especially the case when dealing with (near-) integrated regressors; see, e.g., Cavaliere (2004) and Cavaliere et al. (2010). 
conducting model selection in levels copes with both the stationary and the integrated cases.

2. Regress $y_{t}$ on $\hat{\nu}_{t}$ to obtain $\tilde{y}_{t}$ as regression residuals. From this regression step we also obtain $\hat{\gamma}$, the OLS estimate of $\gamma$.

3. Finally, regress $\tilde{y}_{t}$ on $x_{t-1}$ via IVX and use the provided standard errors (see Equation (12) below) to compute the relevant IVX t-statistic.

From step 3) we thus obtain,

$$
\tilde{\beta}^{i v x}:=\frac{\sum_{t=p+1}^{T} z_{t-1} \tilde{y}_{t}}{\sum_{t=p+1}^{T} z_{t-1} x_{t-1}},
$$

which, upon standardization, is used for inference.

Note that under Assumptions 1 to 4, the standard errors need to take into account two specific features of the data. First, time varying variances are likely to bias the usual standard errors asymptotically. Second, while the estimation error $\left(\hat{v}_{t}-v_{t}\right)$ has no asymptotic effect on the limiting distribution of $\tilde{\beta}^{i v x}$ in the near-integrated context, it does so when $x_{t}$ is covariance stationary. Yet treating the two cases in a different manner is inconvenient since exact knowledge about which is actually the relevant case is typically not available. Consequently, we derive heteroskedasticity-consistent standard errors for the stationary case and show that these are also valid in the near integrated context. In this way, we use the same statistic with the same limiting distribution to cover both cases without having to decide which is which - just like in the original IVX test of Kostakis et al. (2015).

In specific, we use

$$
\operatorname{se}\left(\tilde{\beta}^{i v x}\right):=\sqrt{\frac{\sum_{t=p+1}^{T} z_{t-1}^{2} \tilde{\varepsilon}_{t}^{2}+\hat{\gamma}^{2} \hat{Q}_{T}}{\left(\sum_{t=p+1}^{T} z_{t-1} x_{t-1}\right)^{2}}}
$$

where the finite-sample correction $\hat{Q}_{T}$ used in (12) is given by

$$
\begin{gathered}
\hat{Q}_{T}=\left(\sum_{t=p+1}^{T} z_{t-1} \mathbf{x}_{t-p}^{\prime}\right)\left(\sum_{t=p+1}^{T} \mathbf{x}_{t-p} \mathbf{x}_{t-p}^{\prime}\right)^{-1} \\
\times\left(\sum_{t=p+1}^{T} \mathbf{x}_{t-p} \mathbf{x}_{t-p}^{\prime} \hat{\nu}_{t}^{2}\right)\left(\sum_{t=p+1}^{T} \mathbf{x}_{t-p} \mathbf{x}_{t-p}^{\prime}\right)^{-1}\left(\sum_{t=p+1}^{T} z_{t-1} \mathbf{x}_{t-p}\right)
\end{gathered}
$$


and $\mathbf{x}_{t-p}:=\left(x_{t-1}, \ldots, x_{t-p}\right)^{\prime}$. To compute the White-type standard errors in (12) we make use of the OLS residuals computed from the residual-augmented predictive regression, $\tilde{\varepsilon}_{t}:=\tilde{y}_{t}-\tilde{\beta}^{o l s} x_{t-1}$ where $\tilde{\beta}^{\text {ols }}:=\frac{\sum_{t=2}^{T} x_{t-1} \tilde{y}_{t}}{\sum_{t=2}^{T} x_{t-1}^{2}}$, rather than IVX residuals due to the superconsistency properties of the former in the near-integrated context.

REMARK 6. One may resort to alternative HC variance estimators, e.g., with correction for degrees of freedom (HC1). The HC1 version is obtained here by multiplying the estimated variance by $\frac{T}{T-p-3}$.

REMARK 7. The standard errors in (12) are basically the White standard errors that would have been appropriate under stationarity of $x_{t}$, where the estimation error of $\hat{\nu}_{t}$ does not vanish asymptotically. We show that $\hat{Q}_{T}$ in (12) is dominated under near-integration so that the standard error in (12) is asymptotically equivalent to the one implied by the near-integrated framework, which turns out to be simply $\sqrt{\frac{\sum_{t=p+1}^{T} z_{t-1}^{2} \tilde{\varepsilon}_{t}^{2}}{\left(\sum_{t=p+1}^{T} z_{t-1} x_{t-1}\right)^{2}}}$ as can be seen in Section 3 .

REMARK 8. The near-unit root in $x_{t}$ allows us in principle to use the residuals without the need to use the finite sample correction, but in finite samples the statistics fare better if the correction is included (essentially because, in finite samples, any $|\rho|<1$ is "caught between" stationarity and integration).

\subsection{Extensions to Multiple Predictors}

The discussion so far has side-stepped a couple of aspects relevant for empirical work which we address in this section. They are in fact straightforward extensions of the baseline case and we shall omit some of the technical details.

It is often the case that several predictors are simultaneously considered. Thus, the resulting multiple predictive regression is

$$
y_{t}=\beta^{\prime} \mathbf{x}_{t-1}+u_{t}
$$

where $\mathbf{x}_{t-1}$ follows a $K$-dimensional vector autoregressive data generating process of order $p$, such as,

$$
\begin{aligned}
\mathbf{x}_{t} & =R \mathbf{x}_{t-1}+\mathbf{v}_{t} \\
\mathbf{v}_{t} & =\sum_{j=1}^{p-1} A_{j} \mathbf{v}_{t-j}+\nu_{t}
\end{aligned}
$$

which is either stable or (near) integrated as before depending on the properties of the autoregressive coefficient matrix $R$ ( $\mathbf{v}_{t}$ is taken to be stable in either case). There is endogeneity, possibly in all regressors, expressed as a nonzero coefficient vector in 
the decomposition

$$
u_{t}:=\gamma^{\prime} \nu_{t}+\varepsilon_{t},
$$

and the shocks $\nu_{t}$ and $\varepsilon_{t}$ are heterogeneous, serially independent obeying a multivariate version of Assumption 3.

The implementation of the IVX approach introduced in this paper in the multiple predictive regression case is as follows.

1. Get the vector of residuals $\hat{\nu}_{t}$ from a vector autoregression of order $p$,

$$
\hat{\nu}_{t}:=\mathbf{x}_{t}-\sum_{j=1}^{p} \hat{\Phi}_{j} \mathbf{x}_{t-j}, \quad t=p+1, \ldots, T,
$$

with $\hat{\Phi}_{j}, j=1, \ldots, p$, the matrix of OLS coefficient estimates. Note that the use of AIC (or some other information criteria) in levels, for determining the order $p$, is again recommended.

2. Regress $y_{t}$ on $\hat{\nu}_{t}$ to obtain the adjusted $\tilde{y}_{t}$ as,

$$
\tilde{y}_{t}=y_{t}-\hat{\gamma}^{\prime} \hat{\nu}_{t}
$$

with $\hat{\gamma}$ the OLS estimate of the vector of parameters $\gamma$.

3. Regress $\tilde{y}_{t}$ on $\mathbf{x}_{t-1}$ via IVX with $\mathbf{z}_{t-1}:=(1-\varrho L)_{+}^{-1} \Delta \mathbf{x}_{t-1}$ as instruments to obtain $\tilde{\beta}^{\text {ivx }}$ and use the standard errors provided in Equation (13) below to conduct inference.

The estimated covariance matrix of $\tilde{\beta}^{i v x}$ in this context is given by the familiar "sandwich" formula,

$$
\widehat{\operatorname{Cov}\left(\tilde{\beta}^{i v x}\right)}=B_{T}^{-1} M_{T} B_{T}
$$

where

$$
B_{T}=\sum_{t=2}^{T} \mathbf{z}_{t-1} \mathbf{x}_{t-1}^{\prime}
$$

and

$$
\begin{aligned}
& M_{T}=\sum_{t=2}^{T} \mathbf{z}_{t-1} \mathbf{z}_{t-1}^{\prime} \tilde{\varepsilon}_{t}^{2}+\left(\gamma^{\prime} \otimes\left(\frac{1}{T} \sum_{t=2}^{T} \mathbf{z}_{t-1} \mathbf{x}_{t-p, K}^{\prime}\right)\left(\sum_{t=p+1}^{T} \mathbf{x}_{t-p, K} \mathbf{x}_{t-p, K}^{\prime}\right)^{-1}\right) \times \\
& \times\left(\sum_{t=p+1}^{T} \nu_{t} \nu_{t}^{\prime} \otimes \mathbf{x}_{t-p, K} \mathbf{x}_{t-p, K}^{\prime}\right)\left(\gamma \otimes\left(\sum_{t=p+1}^{T} \mathbf{x}_{t-p, K} \mathbf{x}_{t-p, K}^{\prime}\right)^{-1}\left(\frac{1}{T} \sum_{t=2}^{T} \mathbf{x}_{t-p, K} \mathbf{z}_{t-1}^{\prime}\right)\right)
\end{aligned}
$$

with $\mathbf{x}_{t-p, K}$ corresponding to the vector stacking all $p$ lags of all $K$ regressors, i.e., $\mathbf{x}_{t-p, K}^{\prime}:=\left(x_{t-1,1}, \ldots, x_{t-1, K}, x_{t-2,1}, \ldots, x_{t-2, K}, \ldots, x_{t-p, 1}, \ldots, x_{t-p, K}\right)$.

The limiting distribution of $\tilde{\beta}^{i v x}$ is normal in the stationary case and mixed normal in the near-integrated context; the proofs are simple multivariate extensions 
of the results from the single-regression case so we do not spell them out. More importantly, individual and joint significance tests have their usual standard normal and $\chi^{2}$ limiting distributions irrespective of the persistence and heterogeneity of the DGP as long as the robust covariance matrix estimator in (13) is used.

\section{Asymptotic results}

In this section, we analyze the limiting distributional characteristics of the new reduced-bias IVX tests considering the general framework described in Section 2.4, which also provides us with the results for the simplest case in Section 2.1 as a particular case. We consider two different theoretical frameworks that critically determine the stochastic properties of the predictive variable. On the one hand, we consider stationary predictors, characterized by a fixed coefficient $|\rho|<1$ in (2), and on the other, we allow for near-integration by considering $\rho:=1-\frac{c}{T}$, with $c \geq 0$ and fixed. The main objective of this setting is to acknowledge the uncertainty that researches face regarding the stochastic properties of the predictor, i.e., whether it is stationary or near-integrated when $\hat{\rho}$ is close to, but strictly less than unity in finite samples. This setting includes of course the extreme case of a unit-root when the local parameter $c$ equals zero $(c=0)$.

In the following, we maintain the predictive regression framework in (1) but allow for significant departures from Gaussianity and the restrictive $\operatorname{AR}(1)$ structure for the regressor. We also allow for heterogeneity in the form of time-varying variances, different shapes of the distributions, and even changes in the persistence of the regressor. Financial variables often exhibit time-varying variances in addition to GARCH effects; Kostakis et al. (2015) discuss the GARCH case considering strict stationarity, whereas we relax the i.i.d. assumption in the direction of eliminating weak stationarity via heterogeneity.

Note first that the time-varying properties of the DGP, as stated in Assumptions 1 through 4, imply different behavior in the limit compared to the Gaussian i.i.d. case. In this case, the partial sums of $\nu_{t}$ converge weakly to $M(s):=\int_{0}^{s} \sigma_{\nu}(r) \mathrm{d} W_{v}(r)$, and the partial sums of $\varepsilon_{t}$ to $\int_{0}^{s} \sigma_{\varepsilon}(r) \mathrm{d} W_{\varepsilon}(r)$, with $W_{\varepsilon}$ and $W_{v}$ independent standard Wiener processes; the "classical" case is only recovered when $\sigma_{u}$ and $\sigma_{v}$ are constant. Moreover, the suitably normalized regressor converges weakly to an Ornstein-Uhlenbeck type process driven by the diffusion $M(s)$, i.e.,

$$
\frac{1}{\sqrt{T}} x_{[s T]} \Rightarrow \omega \int_{0}^{s} e^{-r c(r)} \mathrm{d} M(r):=\omega X(s)
$$

where $\omega=\left(1-\sum_{j=1}^{p-1} a_{j}\right)^{-1}$; see, e.g., Cavaliere (2004) for the case with constant $c$.

In the case where $x_{t}$ is stationary, i.e., $|\rho|<1$ and fixed, the following results can be stated. 
Theorem 1. Under Assumptions 1, 2, 3 and 4 i), we have, as $T \rightarrow \infty$, that

$$
\sqrt{T}\left(\tilde{\beta}^{i v x}-\beta\right) \stackrel{d}{\rightarrow} \mathcal{N}\left(0, \sigma_{\beta}^{2}\right)
$$

where

$$
\sigma_{\beta}^{2}:=\frac{\alpha_{0} \int_{0}^{1} \sigma_{v}^{2}(s) \sigma_{\varepsilon}^{2}(s) \mathrm{d} s+\gamma^{2} \alpha_{p}^{\prime} \Omega^{-1} \alpha_{p} \int_{0}^{1} \sigma_{v}^{4}(s) \mathrm{d} s}{\left[\alpha_{0} \int_{0}^{1} \sigma_{v}^{2}(s) \mathrm{d} s\right]^{2}}
$$

with $\alpha_{p}:=\left(\alpha_{0} \ldots \alpha_{p-1}\right)^{\prime}$ and $\Omega:=\left\{\alpha_{|i-j|}\right\}_{1 \leq i, j \leq p}$, where $\alpha_{h}:=\sum b_{j} b_{j+h}$ with $b_{j}$ the moving average coefficients of $x_{t},(1-\rho L)^{-1}\left(1-a_{1} L-\ldots-a_{p-1} L^{p-1}\right)=$ $\sum_{j \geq 0} b_{j} L^{j}$. Furthermore,

$$
\sqrt{T} \operatorname{se}\left(\tilde{\beta}^{i v x}\right) \stackrel{p}{\rightarrow} \sqrt{\sigma_{\beta}^{2}}
$$

and, under the null hypothesis, $H_{0}: \beta=0$,

$$
\tilde{t}_{i v x} \stackrel{d}{\rightarrow} \mathcal{N}(0,1)
$$

The limit behavior changes under near-integration as shown in the following Theorem.

Theorem 2. Under Assumptions 1, 2, 3 and 4ii), we have, as $T \rightarrow \infty$, that

$$
T^{\frac{1}{2}+\frac{\eta}{2}}\left(\tilde{\beta}^{i v x}-\beta\right) \Rightarrow \mathcal{M N}\left(0, \frac{a \int_{0}^{1} \sigma_{\nu}^{2}(s) \sigma_{\varepsilon}^{2}(s) \mathrm{d} s}{2 \omega^{2}\left(X^{2}(1)-\int_{0}^{1} X(s) \mathrm{d} X(s)\right)^{2}}\right)
$$

and

$$
s e\left(\tilde{\beta}^{i v x}\right) \Rightarrow \sqrt{\frac{a}{2 \omega^{2}}} \frac{\sqrt{\int_{0}^{1} \sigma_{\nu}^{2}(s) \sigma_{\varepsilon}^{2}(s) \mathrm{d} s}}{X^{2}(1)-\int_{0}^{1} X(s) \mathrm{d} X(s)}
$$

where $a$ and $\eta$ are fixed, $\omega^{2}$ plays the role of the long-run variance (and is defined in (14)), $X(s)=\int_{0}^{s} e^{-r c(r)} \sigma_{v}(r) \mathrm{d} W_{v}(r)$ and, $\sigma_{\nu}^{2}(s)$ and $\sigma_{\varepsilon}^{2}(s)$ are the variances of $v_{t}$ and $\varepsilon_{t}$, respectively. Moreover, under the null hypothesis, $H_{0}: \beta=0$,

$$
\tilde{t}_{i v x} \Rightarrow \mathcal{N}(0,1) .
$$

The proof of Theorem 2 establishes that $Q_{T}=o_{p}\left(T^{1+\eta}\right)$ so that it is dominated in (12) by $\sum_{t=p+1}^{T} z_{t-1}^{2} \tilde{\varepsilon}_{t}^{2}$ which is of exact order $O_{p}\left(T^{1+\eta}\right)$ (see the Appendix for details), and the residuals estimation effect is negligible in the near-integrated case. The near-integrated case is also more interesting for an evaluation of the local power and for comparison with the original IVX. ${ }^{2}$ The power function of the residual augmented IVX is provided next.

2. The local power in the stationary case is easily derived and we omit the details. 
TheOrem 3. Under Assumptions 1, 2, 3 and 4ii), we have for local alternatives $\beta=\frac{b}{T^{1 / 2+\eta / 2}}$, as $T \rightarrow \infty$ that

$$
\tilde{t}_{i v x} \Rightarrow \mathcal{N}\left(b \sqrt{\frac{2 \omega^{2}}{a}} \frac{X^{2}(1)-\int_{0}^{1} X(s) \mathrm{d} X(s)}{\sqrt{\int_{0}^{1} \sigma_{\nu}^{2}(s) \sigma_{\varepsilon}^{2}(s) \mathrm{d} s}}, 1\right) .
$$

Setting $\omega^{2}=1, \sigma_{v}(s)=\sigma_{v}$ and $\sigma_{\varepsilon}(s)=\sigma_{\varepsilon}$ leads to the results for the particular case studied in Section 2.1.

\section{Finite sample performance}

\subsection{Monte Carlo Setup}

This section compares the two versions of the IVX procedure, the original IVX test which we denote as $t_{i v x}$ and the residual augmented version $\tilde{t}_{i v x}$, with extant procedures under several heterogeneous DGPs. As benchmarks we use the tests of Campbell and Yogo (2006) and of Amihud and Hurvich (2004) and Amihud et al. (2010)

Concretely, we generate $y_{t}$ and $x_{t}$ as in equations (1) and (2), i.e.,

$$
\begin{aligned}
& y_{t}=\beta x_{t-1}+u_{t}, \quad t=2, \ldots, T \\
& x_{t}=\rho x_{t-1}+v_{t} \\
& v_{t}=a_{1} v_{t-1}+e_{t}
\end{aligned}
$$

with $a_{1} \in\{-0.5,0,0.5\}$ and $e_{t} \sim \mathcal{N} i d(0,1)$. We focus on local alternatives of the form $\beta=\frac{b}{T}$ for two sample sizes, $T=200$ and $T=500$. To study the empirical size of the tests we let $b=0$, and for the local power evaluation we consider $b \in\{5,10,15,25\}$, and the persistence of the predictor is controlled by $\rho:=1-\frac{c}{T}$, with $c \in\{0,10,20,40,50\}$. The correlation causing endogeneity is set to -0.95 , which is not an uncommon value in practice; see, e.g., Lewellen (2004).

The efficient tests of Campbell and Yogo (2006) (denoted as $C Y$ ) are analysed, and the residual augmented predictive regression based test of Amihud et al. (2010) (denoted as $A H W$ ) is computed for a fixed $p=2$ to keep complexity under control. In comparison, $t_{i v x}$ does not require specifying the lag length, while for $\tilde{t}_{i v x}$ we chooses $p$ via Akaike's information criteria (AIC). Both $t_{i v x}$ and $\tilde{t}_{i v x}$ are computed by demeaning the dependent variable and the regressor, but not the instrument (see Section 2.5 for details); we also follow Kostakis et al. (2015) and choose $a=1$ and $\eta=0.95$ for the construction of the instruments in both. We employ the proposed standard errors from (12) in the computation of $\tilde{t}_{i v x}$, while, for the classical $t_{i v x}$, we use White standard errors as recommended by Kostakis et al. (2015). We shall also 
consider a version of the original IVX test without White standard errors, denoted by $t_{i v x}^{\#}$, to illustrate the impact of neglected time-varying volatility on the performance of this approach.

The rejection frequencies are computed at the nominal 5\% level based on 10000 Monte Carlo replications, and all results for the $t_{i v x}$ and $\tilde{t}_{i v x}$ tests in Tables $1-4$ are computed based on standard normal critical values.

\subsection{Empirical size and power performance}

Tables 1 and 2 illustrate the empirical size and power properties of the $A H W, C Y$, $t_{i v x}$ and $\tilde{t}_{i v x}$ tests under negative and positive short-run dynamics, i.e., considering (24) with $a_{1}=-0.5$ and $a_{1}=0.5$.

From Table 1, which presents the results obtained when $v_{t}$ follows an AR(1) with $a_{1}=-0.5$ (negative autocorrelation) we observe that when $b=0$ and for the values of $c$ considered that $A H W$ and $t_{i v x}$ are slightly oversized, but that this oversizing decreases as the sample size increases. At the same time, we also observe that $\tilde{t}_{i v x}$ displays slightly conservative behaviour. In this experiment $C Y$ presents the largest size distortions as a consequence of the negative short-run dynamics. This feature of the $C Y$ test has already been noted in the literature; see, e.g., Jansson and Moreira (2006). Note also that in the unit root case $(c=0)$ there are some significant size distortions also for the $t_{i v x}$ and $A H W$ tests. Regarding the empirical power we observe that the $\tilde{t}_{i v x}$ test displays superior power when $c>0$, relative to the other procedures.

In the case of positive short-run dynamics, i.e., when $a_{1}=0.5$ (see Table 2) we observe in general size distortions for all tests, with $t_{i v x}$ displaying the most severe distortions when compared to the other procedures, and $A H W$ and $\tilde{t}_{i v x}$ displaying the smallest distortions.

\subsection{Robustness against empirical features of the data}

To evaluate the performance of the procedures under other empirically relevant features, in Tables 3 and 4 we report results for the empirical size under DGPs with time-varying volatility and time-varying persistence. In specific, we consider five common variance patterns, namely:

1. constant, $\sigma_{\varepsilon}^{2}(s)=\sigma_{\nu}^{2}(s)=1$;

2. an early upward break, $\sigma_{\varepsilon}^{2}(s)=\sigma_{\nu}^{2}(s)=1+8 \mathbb{I}(s>0.3)$;

3. a late upward break, $\sigma_{\varepsilon}^{2}(s)=\sigma_{\nu}^{2}(s)=1+8 \mathbb{I}(s>0.7)$;

4. an early downward break, $\sigma_{\varepsilon}^{2}(s)=\sigma_{\nu}^{2}(s)=9-8 \mathbb{I}(s>0.3)$; and

5. a late downward break, $\sigma_{\varepsilon}^{2}(s)=\sigma_{\nu}^{2}(s)=9-8 \mathbb{I}(s>0.7)$

where $\mathbb{I}($.$) is an indicator function; and to allow for time-varying persistence, we also$ consider 6 patterns for the localization parameter $c$ : 
1. constant close to integration, $c(s)=5$;

2. small break towards stationarity, $c(s)=5+5 \mathbb{I}(s>0.5)$;

3. large break towards stationarity, $c(s)=5+20 \mathbb{I}(s>0.5)$;

4. constant close to stationarity, $c(s)=25$;

5. small break towards integration, $c(s)=25-5 \mathbb{I}(s>0.5)$;

6. large break towards integration, $c(s)=25-20 \mathbb{I}(s>0.5)$.

To gauge the necessity of a correction for time-varying variances, we now compute the IVX test without White heteroskedasticity correction (but note that White standard errors do robustify against time heteroskedasticity, as confirmed by unreported simulations) and denote it by $t_{i v x}^{\#}$; however the $\tilde{t}_{i v x}$ is computed using the standard errors from (12) as before. 


\begin{tabular}{|c|c|c|c|c|c|c|c|c|c|}
\hline & & AHW & $\mathrm{CY}$ & $t_{i v x}$ & $\tilde{t}_{i v x}$ & AHW & $\mathrm{CY}$ & $t_{i v x}$ & $\tilde{t}_{i v x}$ \\
\hline & $b$ & \multicolumn{4}{|c|}{$T=200$} & \multicolumn{4}{|c|}{$T=500$} \\
\hline \multirow{6}{*}{$c=0$} & 0 & 8.9 & 1.1 & 10.6 & 6.30 & 9.4 & 2.5 & 10.4 & 6.3 \\
\hline & 5 & 17.5 & 28.3 & 54.4 & 37.5 & 17.3 & 30.7 & 53.2 & 39.0 \\
\hline & 10 & 67.8 & 94.7 & 93.5 & 86.1 & 65.9 & 97.4 & 93.0 & 87.9 \\
\hline & 15 & 98.2 & 99.4 & 98.9 & 97.3 & 97.8 & 99.8 & 98.7 & 98.1 \\
\hline & 25 & 100.0 & 99.95 & 100.0 & 99.9 & 100.0 & 100.0 & 100.0 & 99.9 \\
\hline & & \multicolumn{4}{|c|}{$T=200$} & \multicolumn{4}{|c|}{$T=500$} \\
\hline \multirow{6}{*}{$c=10$} & 0 & 6.6 & 0.0 & 5.4 & 5.0 & 6.8 & 0.4 & 4.6 & 4.6 \\
\hline & 5 & 8.1 & 0.2 & 13.8 & 14.5 & 7.2 & 2.8 & 12.4 & 14.4 \\
\hline & 10 & 17.1 & 3.8 & 33.2 & 39.6 & 15.0 & 14.8 & 31.0 & 38.7 \\
\hline & 15 & 37.0 & 29.2 & 65.1 & 78.1 & 33.2 & 49.6 & 61.3 & 77.4 \\
\hline & 25 & 96.6 & 94.7 & 96.8 & 99.4 & 95.2 & 98.8 & 96.0 & 99.5 \\
\hline & & \multicolumn{4}{|c|}{$T=200$} & \multicolumn{4}{|c|}{$T=500$} \\
\hline \multirow{6}{*}{$c=20$} & 0 & 6.4 & 0.0 & 4.1 & 4.5 & 6.4 & 0.0 & 4.1 & 4.8 \\
\hline & 5 & 7.1 & 0.0 & 10.4 & 12.3 & 6.4 & 0.2 & 9.4 & 11.1 \\
\hline & 10 & 13.3 & 0.0 & 21.9 & 26.5 & 11.3 & 1.6 & 20.6 & 25.4 \\
\hline & 15 & 24.5 & 0.3 & 40.5 & 50.3 & 19.4 & 7.9 & 37.2 & 47.2 \\
\hline & 25 & 68.8 & 22.6 & 84.2 & 93.9 & 60.4 & 54.3 & 80.1 & 93.2 \\
\hline & & \multicolumn{4}{|c|}{$T=200$} & \multicolumn{4}{|c|}{$T=500$} \\
\hline \multirow{6}{*}{$c=30$} & 0 & 6.0 & 0.0 & 4.3 & 4.9 & 5.8 & 0.0 & 4.0 & 4.9 \\
\hline & 5 & 6.4 & 0.0 & 9.1 & 10.5 & 6.0 & 0.0 & 8.5 & 10.3 \\
\hline & 10 & 11.4 & 0.0 & 17.7 & 21.9 & 9.1 & 0.0 & 15.8 & 20.2 \\
\hline & 15 & 20.1 & 0.0 & 32.4 & 39.3 & 16.1 & 0.5 & 28.4 & 35.9 \\
\hline & 25 & 54.1 & 0.3 & 70.6 & 81.3 & 42.4 & 12.1 & 63.7 & 77.1 \\
\hline & & \multicolumn{4}{|c|}{$T=200$} & \multicolumn{4}{|c|}{$T=500$} \\
\hline \multirow{6}{*}{$c=40$} & 0 & 6.1 & 0.1 & 4.0 & 4.7 & 5.5 & 0.0 & 4.1 & 5.0 \\
\hline & 5 & 6.8 & 0.1 & 8.9 & 10.5 & 5.7 & 0.0 & 7.2 & 9.4 \\
\hline & 10 & 10.5 & 0.1 & 16.8 & 20.0 & 9.1 & 0.0 & 14.3 & 18.3 \\
\hline & 15 & 18.5 & 0.1 & 28.1 & 34.1 & 13.5 & 0.0 & 24.3 & 30.2 \\
\hline & 25 & 45.1 & 0.1 & 60.8 & 71.4 & 34.9 & 0.8 & 52.5 & 65.2 \\
\hline & & \multicolumn{4}{|c|}{$T=200$} & \multicolumn{4}{|c|}{$T=500$} \\
\hline \multirow{5}{*}{$c=50$} & 0 & 5.9 & 0.1 & 3.6 & 4.4 & 5.5 & 0.0 & 3.7 & 5.0 \\
\hline & 5 & 6.5 & 0.1 & 7.8 & 9.7 & 6.2 & 0.0 & 7.1 & 9.5 \\
\hline & 10 & 10.4 & 0.1 & 15.3 & 19.4 & 8.1 & 0.0 & 12.5 & 16.5 \\
\hline & 15 & 16.6 & 0.1 & 26.4 & 32.1 & 12.1 & 0.0 & 20.5 & 26.3 \\
\hline & 25 & 41.6 & 0.1 & 55.5 & 64.9 & 30.2 & 0.0 & 45.1 & 56.3 \\
\hline
\end{tabular}

Notes: AHW denotes the (2-sided) Amihud, Hurwich and Wang test with lag length $p=2$; CY denotes the Campbell and Yogo test, $t_{i v x}$ is IVX test computed following

Kostakis et al. (2015) and $\tilde{t}_{i v x}$ the residual-augmented IVX test procedure, all with maximal lag length $p=\left[4(T / 100)^{0.25}\right]$. The DGP is as in (1) and (2) with $\rho=1-\frac{c}{T}$ and $\beta=\frac{b}{T}$. For further details see the text.

TABLE 1. Size and power against local alternatives, negative short-run AR parameter 


\begin{tabular}{|c|c|c|c|c|c|c|c|c|c|}
\hline & & AHW & CY & $t_{i v x}$ & $\tilde{t}_{i v x}$ & AHW & CY & $t_{i v x}$ & $\tilde{t}_{i v x}$ \\
\hline & $b$ & \multicolumn{4}{|c|}{$T=200$} & \multicolumn{4}{|c|}{$T=500$} \\
\hline \multirow{6}{*}{$c=0$} & 0 & 6.5 & 4.6 & 11.1 & 6.6 & 6.3 & 4.1 & 10.6 & 6.3 \\
\hline & 5 & 94.7 & 100.0 & 98.4 & 96.1 & 95.7 & 100.0 & 98.5 & 97.6 \\
\hline & 10 & 100.0 & 100.0 & 100.0 & 99.9 & 100.0 & 100.0 & 100.0 & 100.0 \\
\hline & 15 & 100.0 & 100.0 & 100.0 & 100.0 & 100.0 & 100.0 & 100.0 & 100.0 \\
\hline & 25 & 100.0 & 100.0 & 100.0 & 100.0 & 100.0 & 100.0 & 100.0 & 100.0 \\
\hline & & \multicolumn{4}{|c|}{$T=200$} & \multicolumn{4}{|c|}{$T=500$} \\
\hline \multirow{6}{*}{$c=10$} & 0 & 6.3 & 4.1 & 8.7 & 5.7 & 6.5 & 3.7 & 8.6 & 6.2 \\
\hline & 5 & 26.5 & 64.4 & 79.0 & 72.9 & 27.3 & 66.0 & 79.9 & 74.9 \\
\hline & 10 & 99.5 & 100.0 & 100.0 & 99.7 & 99.6 & 100.0 & 100.0 & 99.9 \\
\hline & 15 & 100.0 & 100.0 & 100.0 & 100.0 & 100.0 & 100.0 & 100.0 & 100.0 \\
\hline & 25 & 100.0 & 100.0 & 100.0 & 100.0 & 100.0 & 100.0 & 100.0 & 100.0 \\
\hline & & \multicolumn{4}{|c|}{$T=200$} & \multicolumn{4}{|c|}{$T=500$} \\
\hline \multirow{6}{*}{$c=20$} & 0 & 5.7 & 3.1 & 7.2 & 5.6 & 5.9 & 3.1 & 7.5 & 5.9 \\
\hline & 5 & 16.4 & 28.6 & 48.7 & 43.9 & 16.4 & 31.6 & 49.2 & 44.5 \\
\hline & 10 & 70.2 & 94.4 & 98.8 & 97.7 & 74.9 & 96.7 & 99.3 & 98.7 \\
\hline & 15 & 100.0 & 100.0 & 100.0 & 100.0 & 96.0 & 100.0 & 100.0 & 100.0 \\
\hline & 25 & 100.0 & 100.0 & 100.0 & 100.0 & 100.0 & 100.0 & 100.0 & 100.0 \\
\hline & & \multicolumn{4}{|c|}{$T=200$} & \multicolumn{4}{|c|}{$T=500$} \\
\hline \multirow{6}{*}{$c=30$} & 0 & 6.0 & 2.2 & 7.2 & 5.9 & 5.8 & 2.5 & 7.1 & 5.6 \\
\hline & 5 & 13.3 & 16.1 & 35.6 & 32.3 & 13.2 & 18.7 & 37.2 & 34.1 \\
\hline & 10 & 47.6 & 63.2 & 86.8 & 85.4 & 50.5 & 72.9 & 89.8 & 89.2 \\
\hline & 15 & 94.1 & 98.2 & 100.0 & 99.9 & 97.0 & 99.6 & 100.0 & 100.0 \\
\hline & 25 & 100.0 & 100.0 & 100.0 & 100.0 & 100.0 & 100.0 & 100.0 & 100.0 \\
\hline & & \multicolumn{4}{|c|}{$T=200$} & \multicolumn{4}{|c|}{$T=500$} \\
\hline \multirow{6}{*}{$c=40$} & 0 & 5.5 & 1.6 & 6.7 & 5.5 & 5.2 & 1.8 & 6.5 & 5.5 \\
\hline & 5 & 10.2 & 10.4 & 28.4 & 26.5 & 11.0 & 12.2 & 29.7 & 27.4 \\
\hline & 10 & 35.7 & 40.2 & 71.9 & 70.2 & 38.5 & 50.3 & 76.3 & 75.0 \\
\hline & 15 & 79.5 & 82.8 & 98.4 & 98.3 & 84.4 & 91.8 & 99.2 & 99.2 \\
\hline & 25 & 100.0 & 99.9 & 100.0 & 100.0 & 100.0 & 100.0 & 100.0 & 100.0 \\
\hline & & \multicolumn{4}{|c|}{$T=200$} & \multicolumn{4}{|c|}{$T=500$} \\
\hline \multirow{5}{*}{$c=50$} & 0 & 6.1 & 1.3 & 6.6 & 5.7 & 5.3 & 1.4 & 6.7 & 5.7 \\
\hline & 5 & 9.7 & 7.2 & 24.7 & 22.9 & 9.5 & 8.7 & 25.9 & 24.5 \\
\hline & 10 & 28.1 & 26.8 & 61.0 & 59.0 & 30.4 & 33.8 & 64.9 & 63.3 \\
\hline & 15 & 64.3 & 62.3 & 93.0 & 92.7 & 71.2 & 75.7 & 95.9 & 95.7 \\
\hline & 25 & 99.9 & 99.1 & 100.0 & 100.0 & 100.0 & 100.0 & 100.0 & 100.0 \\
\hline
\end{tabular}

Note: See Table 1.

TABLE 2. Size and power against local alternatives, positive short-run AR parameter 
Tables 3 and 4 confirm the conclusions obtained under the homogenous DGPs: the size control of $\tilde{t}_{i v x}$ is overall quite good, and the White-type standard errors account well for time-varying variances (unreported simulations show that not employing the White-type standard errors for the $\tilde{t}_{i v x}$ test under time-varying variances leads to size distortions similar to those of the $t_{i v x}^{\#}$ test). IVX without robust standard errors can be oversized, which was expected; the worst effect is observed for late upward breaks in the variance. AHW exhibits a similar pattern, to an even larger extent. We note that breaks in the persistence parameter $c$ tend to rather have a dampening effect, if any. CY is severely undersized, in line with the previous experiments for negative short-run correlation. For positive short-run correlation, CY now controls size fairly well except for late upward and early downward breaks in the variance; the other three tests do not appear to be sensitive to the sign of the short-run serial correlation of the predictor. The effects are practically the same for both sample sizes, indicating that the size distortions are not finite-sample in nature. 


\begin{tabular}{|c|c|c|c|c|c|c|c|c|c|}
\hline & & AHW & $\mathrm{CY}$ & $t_{i v x}^{\#}$ & $\tilde{t}_{i v x}$ & AHW & $\mathrm{CY}$ & $t_{i v x}^{\#}$ & $\tilde{t}_{i v x}$ \\
\hline$c$ & Var & \multicolumn{4}{|c|}{$T=200$} & \multicolumn{4}{|c|}{$T=500$} \\
\hline \multirow{6}{*}{ const small } & const & 7.6 & 0.1 & 9.4 & 5.7 & 7.4 & 1.2 & 9.7 & 6.2 \\
\hline & early up & 11.5 & 0.1 & 13.2 & 6.4 & 11.2 & 1.6 & 13.8 & 6.5 \\
\hline & late up & 24.1 & 0.6 & 19.1 & 6.6 & 25.2 & 3.9 & 19.6 & 6.3 \\
\hline & early down & 21.5 & 0.4 & 15.0 & 5.7 & 22.1 & 3.0 & 17.1 & 6.0 \\
\hline & late down & 10.7 & 0.4 & 11.9 & 6.1 & 11.1 & 2.3 & 12.4 & 5.9 \\
\hline & & \multicolumn{4}{|c|}{$T=200$} & \multicolumn{4}{|c|}{$T=500$} \\
\hline \multirow{6}{*}{ up small } & const & 7.0 & 0.0 & 8.4 & 5.5 & 7.3 & 0.7 & 9.5 & 5.8 \\
\hline & early up & 11.7 & 0.0 & 12.4 & 6.2 & 11.5 & 1.5 & 12.6 & 6.0 \\
\hline & late up & 23.2 & 0.1 & 16.9 & 5.5 & 24.1 & 2.3 & 17.3 & 5.3 \\
\hline & early down & 22.2 & 0.2 & 15.0 & 6.2 & 22.2 & 2.8 & 16.2 & 6.1 \\
\hline & late down & 10.9 & 0.1 & 11.0 & 6.3 & 11.3 & 1.8 & 12.0 & 6.6 \\
\hline & & \multicolumn{4}{|c|}{$T=200$} & \multicolumn{4}{|c|}{$T=500$} \\
\hline \multirow{6}{*}{ up large } & const & 6.6 & 0.0 & 7.8 & 5.7 & 6.8 & 0.3 & 8.5 & 5.7 \\
\hline & early up & 10.9 & 0.0 & 10.8 & 5.4 & 11.5 & 0.3 & 10.8 & 5.4 \\
\hline & late up & 21.5 & 0.0 & 13.2 & 4.8 & 21.5 & 0.3 & 14.2 & 4.9 \\
\hline & early down & 22.3 & 0.2 & 15.0 & 7.0 & 22.8 & 2.7 & 17.6 & 7.3 \\
\hline & late down & 11.6 & 0.0 & 10.2 & 5.8 & 11.3 & 1.1 & 11.3 & 6.4 \\
\hline & & \multicolumn{4}{|c|}{$T=200$} & \multicolumn{4}{|c|}{$T=500$} \\
\hline \multirow{6}{*}{ const large } & const & 6.2 & 0.0 & 5.1 & 4.9 & 5.6 & 0.0 & 6.4 & 5.2 \\
\hline & early up & 10.6 & 0.0 & 9.7 & 5.4 & 10.4 & 0.0 & 11.0 & 6.0 \\
\hline & late up & 24.4 & 0.1 & 15.8 & 6.6 & 24.5 & 0.1 & 16.3 & 6.2 \\
\hline & early down & 24.0 & 0.0 & 10.8 & 5.9 & 23.2 & 0.0 & 13.5 & 6.1 \\
\hline & late down & 11.1 & 0.0 & 7.80 & 5.6 & 11.0 & 0.0 & 8.4 & 5.5 \\
\hline & & \multicolumn{4}{|c|}{$T=200$} & \multicolumn{4}{|c|}{$T=500$} \\
\hline \multirow{6}{*}{ down small } & const & 6.1 & 0.0 & 6.0 & 5.4 & 6.1 & 0.0 & 7.1 & 5.8 \\
\hline & early up & 10.9 & 0.0 & 10.5 & 5.8 & 11.1 & 0.1 & 11.5 & 6.2 \\
\hline & late up & 23.6 & 0.1 & 16.0 & 6.3 & 23.9 & 0.2 & 17.7 & 6.5 \\
\hline & early down & 23.4 & 0.0 & 10.6 & 5.7 & 23.2 & 0.1 & 13.2 & 6.1 \\
\hline & late down & 10.6 & 0.0 & 7.3 & 5.1 & 10.8 & 0.0 & 9.1 & 5.7 \\
\hline & & \multicolumn{4}{|c|}{$T=200$} & \multicolumn{4}{|c|}{$T=500$} \\
\hline \multirow{5}{*}{ down large } & const & 7.0 & 0.0 & 7.6 & 5.3 & 7.4 & 0.2 & 8.4 & 5.4 \\
\hline & early up & 11.2 & 0.1 & 11.9 & 5.8 & 11.4 & 1.3 & 13.1 & 6.5 \\
\hline & late up & 25.0 & 0.4 & 19.5 & 6.9 & 25.4 & 4.3 & 20.8 & 7.1 \\
\hline & early down & 21.3 & 0.0 & 10.8 & 4.8 & 21.3 & 0.2 & 11.5 & 4.7 \\
\hline & late down & 10.2 & 0.0 & 8.7 & 4.9 & 10.3 & 0.3 & 9.2 & 4.6 \\
\hline
\end{tabular}

Notes: AHW denotes the (2-sided) Amihud, Hurwich and Wang test with lag length $p=2$; CY denotes the Campbell and Yogo test, $t_{i v x}^{\#}$ is IVX test computed following Kostakis et al. (2015) but without White correction, and $\tilde{t}_{i v x}$ is the residual-augmented IVX test procedure, all with maximal lag length $p=\left[4(T / 100)^{0.25}\right]$. The DGP is as in (1) and (2) with $\rho=1-\frac{c_{t}}{T}$ and $\beta=\frac{b}{T}$ and exhibits time-varying variance. For further details see the text.

TABLE 3. Size under breaks in variance and persistence, negative short-run AR parameter 


\begin{tabular}{|c|c|c|c|c|c|c|c|c|c|}
\hline & & AHW & $\mathrm{CY}$ & $t_{i v x}^{\#}$ & $\tilde{t}_{i v x}$ & AHW & $\mathrm{CY}$ & $t_{i v x}^{\#}$ & $\tilde{t}_{i v x}$ \\
\hline$c$ & Var & \multicolumn{4}{|c|}{$T=200$} & \multicolumn{4}{|c|}{$T=500$} \\
\hline \multirow{6}{*}{ const small } & const & 6.6 & 4.5 & 11.4 & 6.8 & 6.3 & 4.2 & 10.9 & 6.6 \\
\hline & early up & 10.0 & 6.8 & 14.0 & 6.7 & 10.0 & 6.6 & 14.0 & 6.6 \\
\hline & late up & 22.6 & 10.4 & 20.3 & 6.6 & 23.4 & 9.6 & 20.5 & 6.7 \\
\hline & early down & 19.9 & 9.0 & 17.3 & 5.7 & 19.9 & 8.4 & 17.8 & 6.0 \\
\hline & late down & 9.8 & 6.7 & 12.8 & 6.3 & 9.5 & 6.1 & 12.4 & 5.7 \\
\hline & & \multicolumn{4}{|c|}{$T=200$} & \multicolumn{4}{|c|}{$T=500$} \\
\hline \multirow{6}{*}{ up small } & const & 5.8 & 4.3 & 10.3 & 6.1 & 6.4 & 4.2 & 9.9 & 5.9 \\
\hline & early up & 10.2 & 6.4 & 13.9 & 7.0 & 10.1 & 6.1 & 13.3 & 6.4 \\
\hline & late up & 21.7 & 8.7 & 18.0 & 6.0 & 21.9 & 8.4 & 18.3 & 5.9 \\
\hline & early down & 19.9 & 9.3 & 18.5 & 7.3 & 19.7 & 9.4 & 17.8 & 6.6 \\
\hline & late down & 9.7 & 7.1 & 12.5 & 6.6 & 9.7 & 6.6 & 12.6 & 7.0 \\
\hline & & \multicolumn{4}{|c|}{$T=200$} & \multicolumn{4}{|c|}{$T=500$} \\
\hline \multirow{6}{*}{ up large } & const & 5.9 & 4.1 & 9.7 & 6.3 & 5.9 & 3.7 & 9.7 & 6.6 \\
\hline & early up & 9.9 & 5.7 & 12.1 & 6.1 & 10.4 & 5.2 & 11.6 & 5.7 \\
\hline & late up & 20.5 & 6.1 & 15.0 & 5.6 & 20.7 & 5.9 & 14.7 & 5.3 \\
\hline & early down & 20.9 & 9.7 & 19.0 & 8.0 & 20.6 & 10.4 & 19.0 & 7.6 \\
\hline & late down & 10.1 & 7.1 & 13.1 & 7.4 & 10.4 & 6.7 & 12.4 & 7.0 \\
\hline & & \multicolumn{4}{|c|}{$T=200$} & \multicolumn{4}{|c|}{$T=500$} \\
\hline \multirow{6}{*}{ const large } & const & 5.8 & 2.6 & 7.5 & 5.6 & 5.6 & 2.8 & 8.1 & 6.0 \\
\hline & early up & 10.9 & 5.3 & 11.4 & 6.2 & 10.5 & 5.4 & 11.7 & 6.6 \\
\hline & late up & 22.7 & 8.0 & 17.6 & 7.0 & 24.1 & 9.1 & 17.9 & 7.0 \\
\hline & early down & 23.1 & 4.5 & 15.5 & 7.2 & 22.3 & 5.6 & 15.8 & 6.4 \\
\hline & late down & 10.7 & 3.8 & 10.4 & 6.3 & 10.1 & 4.1 & 9.9 & 5.7 \\
\hline & & \multicolumn{4}{|c|}{$T=200$} & \multicolumn{4}{|c|}{$T=500$} \\
\hline \multirow{6}{*}{ down small } & const & 5.9 & 2.9 & 8.0 & 5.8 & 6.0 & 3.0 & 8.2 & 5.6 \\
\hline & early up & 10.5 & 5.5 & 12.2 & 6.6 & 10.7 & 5.6 & 12.6 & 6.6 \\
\hline & late up & 23.3 & 8.8 & 18.6 & 7.0 & 24.2 & 9.7 & 18.7 & 6.8 \\
\hline & early down & 22.2 & 4.7 & 15.2 & 7.0 & 21.8 & 5.6 & 15.8 & 6.3 \\
\hline & late down & 10.1 & 3.9 & 10.6 & 6.5 & 10.3 & 4.3 & 9.9 & 5.9 \\
\hline & & \multicolumn{4}{|c|}{$T=200$} & \multicolumn{4}{|c|}{$T=500$} \\
\hline \multirow{5}{*}{ down large } & const & 6.3 & 3.9 & 9.5 & 5.9 & 6.2 & 3.6 & 9.1 & 5.5 \\
\hline & early up & 10.3 & 7.1 & 14.1 & 7.0 & 11.0 & 6.7 & 13.7 & 6.6 \\
\hline & late up & 25.0 & 12.8 & 22.0 & 7.6 & 24.8 & 12.7 & 21.7 & 7.3 \\
\hline & early down & 20.6 & 4.4 & 12.5 & 5.1 & 19.8 & 4.5 & 13.5 & 5.3 \\
\hline & late down & 9.7 & 4.7 & 10.7 & 5.3 & 9.6 & 4.2 & 10.8 & 5.2 \\
\hline
\end{tabular}

Note: See Table 3.

TABLE 4. Size under breaks in variance and persistence, positive short-run AR parameter 


\section{Excess return predictability}

The objective of this empirical part is to re-examine the predictive power of several variables used in Welch and Goyal (2008), updated with information up to December $2013 .^{3}$ using the approaches discussed in the previous sections. We look at the claims by Welch and Goyal (2008) that "evidence suggests that most models are unstable or even spurious" and that "models are no longer significant even in-sample."

\subsection{Background}

According to the findings of Welch and Goyal (2008), most predictive models have performed poorly in sample over the last 30 years. As they argue for many models any earlier apparent statistical significance was often based exclusively on years up to and especially on the years of the Oil shock 1973-1975 (Welch and Goyal 2008, p. 1456).

Ang and Bekaert (2007), considering a sample from 1935 to 2001, report results for several subsamples and for the full sample. Since interest rate data is hard to interpret before the 1951 Treasury Accord, Ang and Bekaert (2007) (as well as Lewellen 2004) consider 1952 as their starting date. Furthermore, Ang and Bekaert (2007) also indicate that the majority of studies establish strong evidence of predictability when data before or up to the early 1990s is used. For instance, Lettau and Ludvigsson (2001) and Goyal and Welch (2003) point out that the predictive power of the dividend yield weakens with the addition of the 1990s decade.

Several researchers suggest that the disapearance of stock return predictability is due to parameter instability or structural breaks and identify the disapearance around 1991 (see, e.g., Pesaran and Timmermann 2002; and Lettau and Nieuwerburgh 2008). A related hypothesis is that predictability was arbitraged away once discovered, in a scenario similar to the attenuation of the January effect. Welch and Goyal (2008) argue that predictability has not been significant in- or out-of-sample over the past 30 years. Still others take a more drastic view and argue that it was never actually there (e.g., Bossaerts and Hillion 1999 and Goyal and Welch 2003).

Henkel et al. (2011) reveal that predictability is a phenomenon whose strength is distinctively time-varying. The dividend yield and commonly used term structure variables are effective predictors almost exclusively during recessions. According to these authors, the robust prominence of busines cycles in these results suggests a potentially substantial tie to the literature on the dynamics of expected returns. Campbell and Cochrane (1999), Menzly et al. (2004) and Bekaert et al. (2009) show that risk premiums are countercyclical and that the time series behaviour of risk premium is higher during recessions.

3. We thank A. Goyal for making this data available on his Web site. 
Since a time-varying predictive relation is the byproduct of the interacting dynamics of expected returns and of the predictors, the complex behaviour of the predictors themselves must be considered when testing for predictability. The underlying fundamentals are the potential micro-level objectives of firms and central banks whose activities jointly determine aggregate predictor variables. The business cycle is an important driver of these micromotives and this lead Henkel et al. (2011) to re-examine predictability using a regime-switching framework capable of matching the time-varying dynamics of predictors to the dynamics of expected returns. It is found that predictors are less persistent and more volatile during recessions. Several features of their analysis stand out: the random walk model of stock prices prevailed in the 1970s based on CRSP data from the 1960s era expansion; predictability emerged in research of the late 1970s and mid-1980s, following several recessions; and predictability was subsequently doubted following the long booms of the 1980s and 1990s.

Hence, in line with Ang and Bekaert (2007) and given the availability of data, we revisit the impact of the addition of the 1990s first, followed by the analysis of the effects of adding the period from January 2000 to September 2007 and finally the remaining sample period (October 2007 to December 2013). Moreover, in order to remove the possible impact of the Oil shock (1973-1975) we repeat the analysis starting in 1976.

Given the available empirical evidence of change in strength of predictability of some variables over time, in what follows we split the sample into eigth periods. These changes appear to be accompanied by changes in the persistence of the considered regressors. ${ }^{4}$ In particular, we consider the eight time periods: i) Jan 1952 - Dec 1989; ii) Jan 1952 - Dec 1999; iii) Jan 1952 - Sep 2007; iv) Jan 1952 - Dec 2013; v) Jan 1976 - Dec 1989; vi) Jan 1976 - Dec 1999; vii) Jan 1976 - Sep 2007; and viii) Jan 1976 - Dec 2013.

\subsection{Data}

The dependent variable is the equity premium (or excess return), i.e., the total rate of return on the stock market minus the prevailing short-term interest rate. Stock returns are the continuously compounded returns on the S\&P 500 index, including dividends, and the risk-free rate is the Treasury-bill rate.

The independent variables used are: i) the 12-month moving sums of dividends (D12) paid on the S\&P 500 index; ii) the dividend price-ratio (d/p) computed as the difference between the $\log$ of dividends and the log of prices; iii) the dividend yield $(\mathrm{d} / \mathrm{y})$ computed as the difference between the $\log$ of dividends and the $\log$ of lagged prices; iv) the 12-month moving sums of earnings on the S\&P 500 index

4. See the results in Appendix B for more details. 
$(\mathrm{E} 12) ; \mathrm{v})$ the earnings price-ratio (e/p) computed as the difference between the log of earnings and the log of prices; vi) the dividend payout-ratio (d/e) computed as the difference between the log of dividends and the log of earnings; vii) the stock variance (svar) computed as the sum of squared daily returns on the S\&P 500; viii) the crosssectional beta premium (csp) which measures the relative valuations of high- and low-beta stocks; ix) the book-to-market ratio $(\mathrm{b} / \mathrm{m})$ computed as the ratio of book value to market value for the Dow Jones industrial average. To include corporate issuing activity we also use $\mathrm{x}$ ) the net equity expansion (ntis) computed as the ratio of 12-month moving sums of net issues by NYSE listed stocks divided by the total end-of-year market capitalization of NYSE stocks; and xi) the percent equity issuing (eqis), which is the ratio of equity issuing activity as a fraction of total issuing activity.

A further set of predictors used is: the treasury bills (tbl) rates; the long term government bond yield (lty); the term spread (tms) which is the difference between the long term yield on government bonds and the treasury-bill; the default yield spread (dfy) which is the difference between BAA and AAA-rated corporate bond yields. The default return spread (dfr) is the difference between long-term corporate bond and long-term government bond returns; inflation (infl) which corresponds to the consumer price index (all urban consumers); and long-term government bond returns (ltr). For details on the construction of these variables and for a greater description see Welch and Goyal (2008).

\subsection{Findings}

Tables 5 and 6 report the predictability test results computed from $t_{i v x}, \tilde{t}_{i v x}$ and the OLS based tests procedures over four subperiods of analysis starting in January 1952. From Table 5 it is interesting to observe that the OLS based test procedure finds most evidence of predictability in the subsample from January 1952 to December 1989, and as we add information the number of significant predictors decreases. Note that in the subsample from January 1952 to December 1989, based on this procedure, nine variables (d/p, d/y, d/e, tbl, tms, ntis, infl, ltr, svar) seemed to be significant; whereas in the following subperiods (January 1952 to December 1999; to September 2007, and to December 2013) the number of significant variables reduced to six (tbl, lty, tms, ntis, infl, ltr), to two (infl, ltr) and increases again to six (tbl, lty, tms, infl, ltr, svar), respectively. However, if we look at the results obtained with the two IVX approaches, the number of significant predictors is smaller. The original IVX approach for the four periods under analysis (January 1952 to December 1989; January 1952 to December 1999; January 1952 to September 2007 and January 1952 to December 2013) finds 5, 5, 2 and 4 significant predictors, respectively; whereas the residual augmented IVX approach proposed in this paper finds 5, 6, 2 and 5, respectively. 


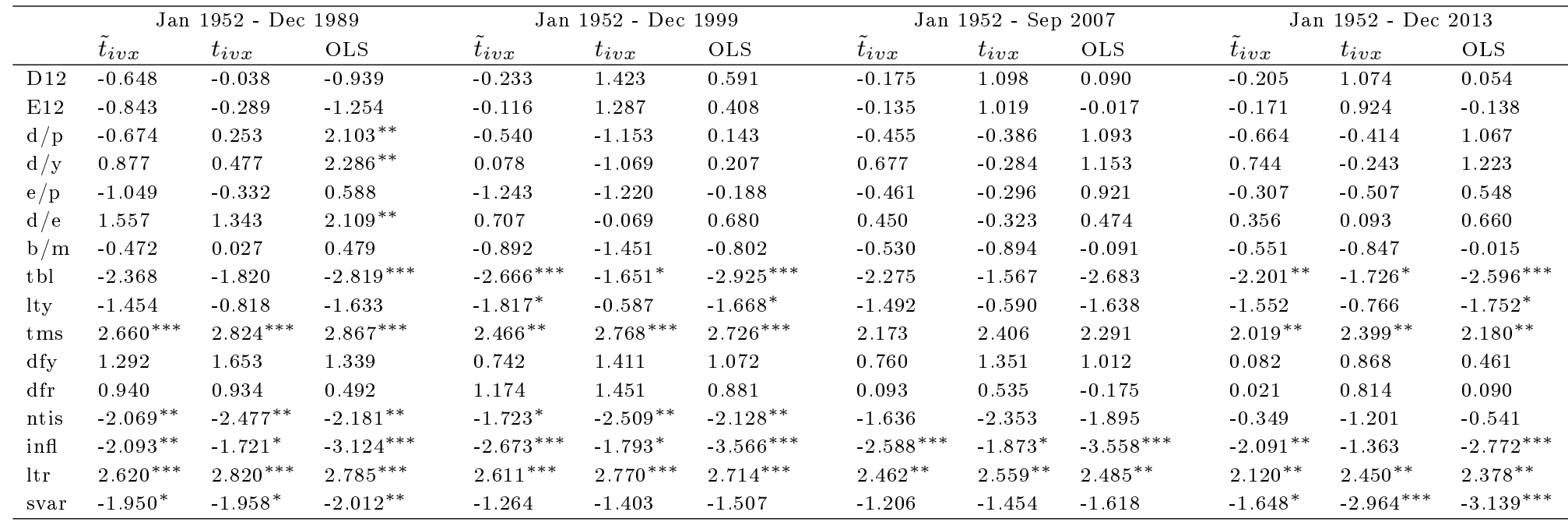

TABLE 5. Testing for Predictability (starting date January 1952) 


\begin{tabular}{|c|c|c|c|c|c|c|c|c|c|c|c|c|}
\hline & \multicolumn{3}{|c|}{ Jan 1975 - Dec 1989} & \multicolumn{3}{|c|}{ Jan 1975 - Dec 1999} & \multicolumn{3}{|c|}{ Jan 1975 - Sep 2007} & \multicolumn{3}{|c|}{ Jan 1975 - Dec 2013} \\
\hline & $\tilde{t}_{i v x}$ & $t_{i v x}$ & OLS & $\tilde{t}_{i v x}$ & $t_{i v x}$ & OLS & $\tilde{t}_{i v x}$ & $t_{i v x}$ & OLS & $\tilde{t}_{i v x}$ & $t_{i v x}$ & OLS \\
\hline D12 & -0.191 & 0.311 & 0.156 & -0.062 & $1.854^{*}$ & 1.358 & 0.229 & 1.437 & 0.578 & 0.038 & 1.271 & 0.340 \\
\hline E12 & -0.315 & 0.055 & -0.170 & 0.618 & $1.996^{* *}$ & 1.450 & 0.431 & 1.489 & 0.605 & 0.154 & 1.128 & 0.183 \\
\hline $\mathrm{d} / \mathrm{p}$ & -0.302 & 0.668 & 0.939 & -0.393 & -1.475 & -0.774 & -0.260 & -0.416 & 0.581 & -0.467 & -0.417 & 0.621 \\
\hline$d / y$ & 0.599 & 0.632 & 1.077 & -0.288 & -1.535 & -0.792 & 0.456 & -0.495 & 0.589 & 0.534 & -0.407 & 0.739 \\
\hline $\mathrm{e} / \mathrm{p}$ & -0.455 & 0.087 & 0.332 & -0.987 & -1.301 & -0.616 & -0.021 & -0.261 & 0.754 & -0.101 & -0.482 & 0.415 \\
\hline$d / e$ & 0.591 & 0.852 & 0.795 & -0.696 & -0.031 & -0.275 & -0.512 & -0.349 & -0.309 & -0.069 & 0.194 & 0.204 \\
\hline $\mathrm{b} / \mathrm{m}$ & 0.103 & 0.040 & 0.300 & -0.415 & -1.609 & -0.955 & -0.189 & -1.088 & -0.145 & -0.181 & -1.022 & -0.036 \\
\hline tbl & $-1.892^{*}$ & -1.451 & -1.506 & $-2.273^{* *}$ & $-2.002^{* *}$ & $-1.973^{* *}$ & -1.520 & -1.628 & -1.273 & -1.242 & $-1.712^{*}$ & -1.230 \\
\hline lty & -1.431 & -0.863 & -0.935 & $-2.084^{* *}$ & $-1.746^{*}$ & $-1.778^{*}$ & -1.277 & -1.375 & -0.966 & -1.126 & -1.508 & -0.992 \\
\hline tms & 1.402 & 1.432 & 1.423 & 0.889 & 1.181 & 1.141 & 0.858 & 1.014 & 0.983 & 0.854 & 1.144 & 1.007 \\
\hline dfy & 1.239 & 0.859 & 1.453 & 0.813 & -0.574 & 0.388 & 0.501 & -0.590 & 0.536 & -0.123 & -0.916 & -0.035 \\
\hline dfr & 0.866 & 0.066 & 0.259 & 1.639 & 0.083 & 0.645 & -0.094 & -0.873 & -0.733 & -0.210 & -0.421 & -0.345 \\
\hline ntis & $-2.579^{* * *}$ & $-2.257^{* *}$ & $-2.196^{* *}$ & $-1.997^{* *}$ & -1.368 & -1.627 & $-1.729^{*}$ & -1.416 & -1.543 & 0.021 & -0.076 & -0.057 \\
\hline infl & -1.532 & -1.415 & -1.394 & $-1.879^{*}$ & $-2.513^{* *}$ & $-2.201^{* *}$ & $-1.956^{*}$ & $-2.443^{* *}$ & $-2.074^{* *}$ & -1.035 & $-1.749^{*}$ & -1.234 \\
\hline ltr & 1.415 & 1.285 & 1.428 & 1.536 & 1.027 & 1.389 & 1.382 & 0.922 & 1.279 & 1.217 & 0.949 & 1.300 \\
\hline svar & $-2.174^{* *}$ & -1.628 & -1.584 & -1.105 & -1.477 & -1.237 & -1.112 & -1.560 & -1.353 & -1.636 & $-3.092^{* * *}$ & $-2.951^{* * *}$ \\
\hline
\end{tabular}

TABle 6. Testing for Predictability (starting date January 1976) 
Performing the same analysis, but starting now in January 1975 instead of January 1952, the OLS based approach finds 1, 3, 1 and 1 significant predictors in the four subsamples under analysis (January 1975 to December 1989; January 1975 to December 1999; January 1975 to September 2007 and January 1976 to December 2013), respectively. Thus, based on this statistic the period between January 1976 to December 1999 is the one which presents more evidence of predictability. Using the IVX based approaches, the number of significant predictors is 1, 5, 1 and 3, for the original IVX and 3, 4, 2 and 0 for the residual augmented IVX approach, for the four subperiods under analysis, respectively. Hence, both IVX based approaches also identify the period between 1976 and 1999 as the period with strongest evidence of predictability.

The results in Table 6 agree to a certain extend with the conclusions put forward by Welch and Goyal (2008) that apparent statistical significance was often based exclusively on years up to and especially on the years of the Oil Shock of 1973-1975.

\section{Conclusions}

This paper introduced a new IVX test statistic computed from a residual augmented predictive regression as considered in Amihud and Hurvich (2004) and reexamined the empirical evidence on predictability of stock returns of Welch and Goyal (2008) using these new robust methods.

To this end we resorted to IVX estimation and testing, and proposed a residualaugmented variant that allows practitioners to distinguish more reliably between the null of no predictability and the alternative. The method is asymptotically correct under near-integration as well as under stationarity of the regressor, has improved local power under high regressor persistence, and allows, e.g., for heterogeneity of the data in the form of time-varying variances.

The results derived here on bias correction can be generalized for other types of instrumental variable estimation than just IVX. The IV framework of Breitung and Demetrescu (2015), who distinguish between type-I instruments that are less persistent than the initial regressor (the IVX instrument is actually of type I; see Breitung and Demetrescu 2015), and type-II instruments that are (stochastically) trending, yet exogenous, allows for a quick discussion: a careful examination of the arguments presented here shows that they are easily extended for type-I instruments, but type-II instruments behave like the OLS estimator where residual-augmentation is not improving on the test procedure even asymptotically.

The provided Monte Carlo evidence shows that the asymptotic improvements are a good indicative of the finite-sample performance. Also, the empirical analysis showed that the bias-adjusted IVX procedure detected predictability more often than the original IVX procedure. 


\section{References}

Amado, C. and T. Teräsvirta (2014). "Modelling changes in the unconditional variance of long stock return series." Journal of Empirical Finance, 25(1), 15-35.

Amado, Cristina and Timo Teräsvirta (2013). "Modelling volatility by variance decomposition." Journal of Econometrics, 175(2), 142-153.

Amihud, Yakov and Clifford M Hurvich (2004). "Predictive regressions: A reduced-bias estimation method." Journal of Financial and Quantitative Analysis, 39(4), 813-841.

Amihud, Yakov, Clifford M Hurvich, and Yi Wang (2009). "Multiple-predictor regressions: Hypothesis testing." Review of Financial Studies, 22(1), 413-434.

Amihud, Yakov, Clifford M Hurvich, and Yi Wang (2010). "Predictive regression with order-p autoregressive predictors." Journal of Empirical Finance, 17(3), $513-525$.

Andrews, D. W. K. and W. Ploberger (1994). "Optimal tests when a nuisance parameter is present only under the alternative." Econometrica, 62, 13831414 .

Andrews, D.W.K (1993). "Tests for parameter instability and structural change with unknown change point." Econometrica, 61, 821-856.

Ang, A. and G. Bekaert (2007). "Return predictability: Is it there?" Review of Financial Studies, 20(3), 651-707.

Avramov, Doron, Laurent Barras, and Robert Kosowski (2010). "Hedge fund predictability under the magnifying glass: The economic value of forecasting individual fund returns." Working paper.

Bali, Turan G (2008). "The intertemporal relation between expected returns and risk." Journal of Financial Economics, 87(1), 101-131.

Bekaert, G., E. Engstrom, and Y. Xing (2009). "Uncertainty, and Asset Prices." Journal of Financial Economics, 91(1), 59-82.

Bossaerts, P. and P. Hillion (1999). "Implementing Statistical Criteria to Select Return Forecasting Models: What do we Learn?" Review of Financial Studies, 12(2), 405-428.

Breitung, J. and M. Demetrescu (2015). "Instrumental Variable and Variable Addition Based Inference in Predictive Regressions." Journal of Econometrics, 187(1), 358-375.

Busetti, F. and A. M. R. Taylor (2004). "Tests of stationarity against a change in persistence." Journal of Econometrics, 123, 33-66. 
Cai, Zongwu and Yunfei Wang (2014). "Corrigendum to "Testing predictive regression models with nonstationary regressors" [J. Econometrics 178 (2014) 4-14]." Journal of Econometrics, 181(2), 194.

Campbell, J. Y. and J. H. Cochrane (1999). "By Force of Habit: A Consumption-Based Explanation of Aggregate Stock Market Behavior." Journal of Political Economy, 107(1), 205-251.

Campbell, J. Y. and M. Yogo (2006). "Efficient Tests of Stock Return Predictability." Journal of Financial Economics, 81(1), 27-60.

Campbell, John Y (2008). "Viewpoint: Estimating the equity premium." Canadian Journal of Economics/Revue canadienne d'économique, 41(1), 121.

Camponovo, Lorenzo (2015). "Differencing Transformations and Inference in Predictive Regression Models." Econometric Theory, 31(6), 1331-1358.

Cavaliere, G. (2004). "Unit Root Tests under Time-Varying Variances." Econometric Reviews, 23(3), 259-292.

Cavaliere, G., A. Rahbek, and A. M. R. Taylor (2010). "Testing for CoIntegration in Vector Autoregressions with Non-Stationary Volatility." Journal of Econometrics, 158(1), 7-24.

Cavanagh, C. L., G. Elliott, and J. H. Stock (1995). "Inference in Models with Nearly Integrated Regressors." Econometric Theory, 11(5), 1131-1147.

Chang, Yoosoon and Joon Y Park (2002). "On the asymptotics of ADF tests for unit roots." Econometric Reviews, 21(4), 431-447.

Chun, Sungju (2009). "Are International Equity Market Returns Predictable?" Boston University working paper.

Davidson, James (1994). Stochastic Limit Theory: An Introduction for Econometricians. Oxford university press.

Davies, R.B. (1977). "Hypothesis testing when a nuisance parameter is present only under the alternative." Biometrika 64, 247-54.

Demetrescu, Matei (2014a). "Corrigendum to "Testing predictive regression models with nonstationary regressors" [J. Econometrics 178 (2014) 4-14]." Working paper, University of Bonn.

Demetrescu, Matei (2014b). "Enhancing the local power of IVX-based tests in predictive regressions." Economics Letters, 124(2), 269-273.

Elliott, G. and J. H. Stock (1994). "Inference in Time Series Regression when the Order of Integration of a Regressor Is Unknown." Econometric Theory, $10(3-4), 672-700$.

Faust, Jon and Jonathan H Wright (2011). "Efficient prediction of excess returns." Review of Economics and Statistics, 93(2), 647-659. 
Gonzalo, Jesús and Jean-Yves Pitarakis (2012). "Regime-Specific Predictability in Predictive Regressions." Journal of Business 83 Economic Statistics, 30(2), 229-241.

Goyal, A. and I. Welch (2003). "Predicting the Equity Premium with Dividend Ratios." Management Science, 49(5), 639-654.

Guidolin, Massimo and Allan Timmermann (2006). "An Econometric Model of Nonlinear Dynamics in the Joint Distribution of Stock and Bond Returns." Journal of Applied Econometrics, 21(1), 1-22.

Hansen, B. E. (1992). "Convergence to Stochastic Integrals for Dependent Heterogeneous Processes." Econometric Theory, 8(4), 489-500.

Hansen, B.E. (1991). "Tests for Parameter Instability in Regressions with I(1) Processes." Journal of Business and Economic Statistics 10, 321-335.

Harvey, D. L., S. J. Leybourne, and A. M. R. Taylor (2006). "Modified tests for a change in persistence." Journal of Econometrics, 134, 441-469.

Henkel, S. J., J. S. Martin, and F. Nardari (2011). "Time-Varying Short-Horizon Predictability." Journal of Financial Economics, 99(3), 560-580.

Jansson, M. and M. J. Moreira (2006). "Optimal Inference in Regression Models with Nearly Integrated Regressors." Econometrica, 74(3), 681-714.

Johannes, M., A. Korteweg, and N. Polson (2014). "Sequential Learning, Predictability, and Optimal Portfolio Returns." The Journal of Finance, 69(2), 611-644.

Kim, J. (2000). "Detection of change in persistence of a linear time series." Journal of Econometrics, 95, 97-116.

Kim, J. Y., J. Belaire-Franch, and R. Amador (2002). "Corringendum to detection of change in persistence of a linear time series." Journal of Econometrics, 109, 389-392.

Kostakis, A., T. Magdalinos, and M. P. Stamatogiannis (2015). "Robust Econometric Inference for Stock Return Predictability." Review of Financial Studies, 28(5), 1506-1553.

Lee, J. (2012). "Predictive Quantile Regressions with persistent covariates." Working paper, Department of Economics, Yale University.

Lettau, M. and S. Ludvigsson (2001). "Consumption, Aggregate Wealth, and Expected Stock Returns." Journal of Finance, 56(3), 815-850.

Lettau, M. and S. Van Nieuwerburgh (2008). "Reconciling the Return Predictability Evidence." The Review of Financial Studies, 21(4), 1607-1652.

Lewellen, Jonathan (2004). "Predicting returns with financial ratios." Journal of Financial Economics, 74(2), 209-235. 
Magdalinos, T. and P. C. B. Phillips (2009). "Limit Theory for Cointegrated Systems with Moderately Integrated and Moderately Explosive Regressors." Econometric Theory, 25(2), 482-526.

Mankiw, Gregory N and Matthew D Shapiro (1986). "Do we reject too often?: Small sample properties of tests of rational expectations models." Economics Letters, 20(2), 139-145.

Maynard, Alex and Katsumi Shimotsu (2009). "Covariance-based orthogonality tests for regressors with unknown persistence." Econometric Theory, 25(1), $63-116$.

Menzly, L., T. Santos, and P. Veronesi (2004). "Understanding Predictability." Journal of Political Economy, 112(1), 1-47.

Park, J. Y. (1990). "Testing for Unit Roots and Cointegration by Variable Addition in T. Fomby and F. Rhodes (eds) Advances in Econometrics: Cointegration, Spurious Regressions and Unit Roots." London Jai Press, 107-134.

Pesaran, M. H. and A. Timmermann (2002). "Market timing and return prediction under model instability." Journal of Empirical Finance, 9(5), 495510.

Phillips, P. C. B. and J. H. Lee (2013). "Predictive regression under various degrees of persistence and robust long-horizon regression." Journal of Econometrics, 177(2), 250-264.

Phillips, P. C. B. and K. L. Xu (2006). "Inference in Autoregression Under Heteroskedasticity." Journal of Time Series Analysis, 27(2), 289-308.

Phillips, Peter C B (2015). "Pitfalls and Possibilities in Predictive Regression." Journal of Financial Econometrics, 13(3), 521-555.

Rodrigues, Paulo M. M. and Antonio Rubia (2011). "A Class of Robust Tests in Augmented Predictive Regressions." Working Paper 26/2011, Banco de Portugal.

Stambaugh, Robert F (1986). "Bias in regressions with lagged stochastic regressors." CRSP Working Paper No 156, University of Chicago.

Stambaugh, Robert F. (1999). "Predictive regressions." Journal of Financial Economics, 54(3), 375-421.

Teräsvirta, Timo and Zhenfang Zhao (2011). "Stylized facts of return series, robust estimates and three popular models of volatility." Applied Financial Economics, 21(1-2), 67-94.

Welch, I. and A. Goyal (2008). "A Comprehensive Look at the Empirical Performance of Equity Premium Prediction." Review of Financial Studies, 21(4), 1455-1508. 


\section{Appendix A: Technical Appendix}

\section{A.1. Preliminary Results}

Throughout the proofs, we consider $\sum_{j=0}^{t-1} \varrho^{k j}=\frac{1-\varrho^{k t}}{1-\varrho^{k}}=\frac{T^{\eta}}{a}\left(\frac{1-\varrho^{k t}}{1+\varrho+\ldots+\varrho^{k-1}}\right) \leq$ $\frac{1}{k a} T^{\eta}$ for large enough $T$ and fixed $k$, where $\varrho:=1-\frac{a}{T^{\eta}}$ with $\eta \in(0,1)$ and $a>0$ and fixed. Furthermore, let $C$ denote a generic constant whose value may change from occurrence to occurrence.

LEMмA A.1. Under the assumptions of Theorem 1, as $T \rightarrow \infty$, it follows that

1. $\frac{1}{T} \sum_{t=p+1}^{T} x_{t-1} \mathbf{x}_{t-p} \stackrel{p}{\rightarrow} \alpha_{p}^{\prime} \int_{0}^{1} \sigma_{v}^{2} \mathrm{~d} s$, where $\alpha_{p}:=\left(\alpha_{0}, \ldots, \alpha_{p-1}\right)$ and $\mathbf{x}_{t-p}:=$ $\left(x_{t-1}, \ldots, x_{t-p}\right)^{\prime}$ and $\alpha_{h}$ is as defined in Theorem 3.1;

2. $\frac{1}{T} \sum_{t=p+1}^{T} \mathbf{x}_{t-p} \mathbf{x}_{t-p}^{\prime} \stackrel{p}{\rightarrow} \boldsymbol{\Omega} \int_{0}^{1} \sigma_{v}^{2} \mathrm{~d} s$, where $\boldsymbol{\Omega}$ is a $p \times p$ matrix with generic element $a_{i j}=\alpha_{|i-j|}$;

3. $\frac{1}{T} \sum_{t=p+1}^{T} \mathbf{x}_{t-p} \mathbf{x}_{t-p}^{\prime} \nu_{t}^{2} \stackrel{p}{\rightarrow} \boldsymbol{\Omega} \int_{0}^{1} \sigma_{v}^{4}(s) \mathrm{d} s ;$

4. $\frac{1}{T} \sum_{t=p+1}^{T} z_{t-1}^{2} \varepsilon_{t}^{2} \stackrel{p}{\rightarrow} \alpha_{0} \int_{0}^{1} \sigma_{v}^{2}(s) \sigma_{\varepsilon}^{2}(s) \mathrm{d} s$.

\section{Proof of Lemma A.1}

Phillips and $\mathrm{Xu}$ (2006) show in their Lemma 1 that $\frac{1}{T} \sum_{t=h+1}^{T} x_{t} x_{t-h} \stackrel{p}{\rightarrow}$ $\alpha_{h} \int_{0}^{1} \sigma_{v}^{2} \mathrm{~d} s, h=0,1, \ldots, p-1$; this suffices to establish the results in the first two items. The result in item 3 also follows directly from Lemma 1 of Phillips and $\mathrm{Xu}$ (2006), and the proof can be adapted in a straightforward manner to establish the result in item 4 .

LemmA A.2. Under the assumptions of Theorem 2, as $T \rightarrow \infty$, it follows that

$$
\frac{\sum_{t=2}^{T} \tilde{z}_{t-1} \varepsilon_{t}}{\sqrt{\sum_{t=2}^{T} \tilde{z}_{t-1}^{2} \varepsilon_{t}^{2}}} \stackrel{d}{\rightarrow} \mathcal{N}(0,1)
$$

where $\tilde{z}_{t}=\sum_{j=0}^{t-1} \varrho^{j} \nu_{t-j}$.

\section{Proof of Lemma A.2}

Consider $s_{T}^{2}:=\frac{1}{T^{1+\eta}} \sum_{t=2}^{T} \sum_{j=0}^{t-2} \varrho^{2 j} \sigma_{\nu, t-1-j}^{2} \sigma_{\varepsilon, t}^{2}$ and note that $s_{T}^{2}$ is bounded and bounded away from zero, since

$\frac{\min _{1 \leq t \leq T} \sigma_{\nu, t}^{2} \min _{1 \leq t \leq T} \sigma_{\varepsilon, t}^{2}}{T^{1+\eta}} \sum_{t=2}^{T} \sum_{j=0}^{t-2} \varrho^{2 j} \leq s_{T}^{2} \leq \frac{\max _{1 \leq t \leq T} \sigma_{\nu, t}^{2} \max _{1 \leq t \leq T} \sigma_{\varepsilon, t}^{2}}{T^{1+\eta}} \sum_{t=2}^{T} \sum_{j=0}^{t-2} \varrho^{2 j}$ 
where $\sum_{t=2}^{T} \sum_{j=0}^{t-2} \varrho^{2 j} \sim C T^{1+\eta}$.

Since,

$$
\frac{\sum_{t=2}^{T} \tilde{z}_{t-1} \varepsilon_{t}}{\sqrt{\sum_{t=2}^{T} \tilde{z}_{t-1}^{2} \varepsilon_{t}^{2}}}=\frac{1}{T^{1 / 2+\eta / 2}} \sum_{t=2}^{T} \frac{\tilde{z}_{t-1} \varepsilon_{t}}{s_{T}} \sqrt{\frac{\sum_{t=2}^{T} \sum_{j=0}^{t-2} \varrho^{2 j} \sigma_{\nu, t-1-j}^{2} \sigma_{\varepsilon, t}^{2}}{\sum_{t=2}^{T} \tilde{z}_{t-1}^{2} \varepsilon_{t}^{2}}}
$$

we show next that $\frac{1}{T^{1 / 2+\eta / 2}} \sum_{t=2}^{T} \frac{\tilde{z}_{t-1} \varepsilon_{t}}{s_{T}}$ follows a limiting standard normal distribution by resorting to a central limit theorem for martingale difference [md] arrays (Davidson 1994, Theorem 24.3). However, to apply it, we need to show that, i) $\max _{t} \frac{1}{T^{1 / 2+\eta / 2}}\left|\frac{\tilde{z}_{t-1} \varepsilon_{t}}{s_{T}}\right| \stackrel{p}{\rightarrow} 0$ and ii) $\frac{1}{T^{1+\eta}} \sum_{t=2}^{T} \frac{\tilde{z}_{t-1}^{2} \varepsilon_{t}^{2}}{s_{T}^{2}} \stackrel{p}{\rightarrow} 1$.

Given that the result in ii) also implies

$$
\sqrt{\frac{\sum_{t=2}^{T} \sum_{j=0}^{t-2} \varrho^{2 j} \sigma_{\nu, t-1-j}^{2} \sigma_{\varepsilon, t}^{2}}{\sum_{t=2}^{T} \tilde{z}_{t-1}^{2} \varepsilon_{t}^{2}}} \stackrel{p}{\rightarrow} 1
$$

hence the result in (A.1) would follow.

To verify i), note that uniform boundedness of moments of order $2+\delta^{*}$ for some $\delta^{*}>0$ of $T^{-\eta / 2} \tilde{z}_{t-1} \varepsilon_{t}$ suffices to establish this condition. An application of Hölder's inequality shows that uniformly bounded 4th order moments of

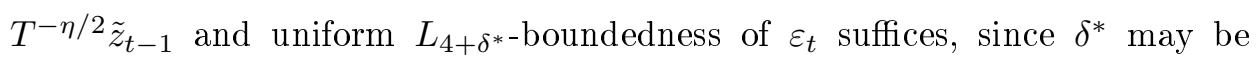
chosen arbitrarily close to zero, so we check the uniform boundedness of

$$
\mathrm{E}\left(\frac{\tilde{z}_{t-1}^{4}}{T^{2 \eta}}\right)=\frac{1}{T^{2 \eta}} \sum_{j=0}^{t-2} \sum_{k=0}^{t-2} \sum_{l=0}^{t-2} \sum_{m=0}^{t-2} \varrho^{j} \varrho^{k} \varrho^{l} \varrho^{m}\left(\nu_{t-j} \nu_{t-k} \nu_{t-l} \nu_{t-m}\right)
$$

Due to the serial independence of $\nu_{t}$, the expectation $\left(\nu_{t-j} \nu_{t-k} \nu_{t-l} \nu_{t-m}\right)$ is nonzero only if the indices are pairwise equal, thus we can simplify (A.3) as,

$$
\mathrm{E}\left(\frac{\tilde{z}_{t-1}^{4}}{T^{2 \eta}}\right)=\frac{1}{T^{2 \eta}} \sum_{j=0}^{t-2} \sum_{k=0}^{t-2} \varrho^{2 j} \varrho^{2 k}\left(\nu_{t-j}^{2} \nu_{t-k}^{2}\right)
$$

Since $\nu_{t}$ is uniformly $L_{4}$-bounded, the expectations on the r.h.s. are uniformly bounded for any $t, k$ and $j$, therefore,

$0 \leq \mathrm{E}\left(\frac{\tilde{z}_{t-1}^{4}}{T^{2 \eta}}\right) \leq C \frac{1}{T^{2 \eta}} \sum_{j=0}^{t-2} \sum_{k=0}^{t-2} \varrho^{2 j} \varrho^{2 k}=C \frac{1}{T^{2 \eta}}\left(\sum_{j=0}^{t-2} \varrho^{2 j}\right)^{2} \leq C \frac{1}{T^{2 \eta}}\left(\sum_{j=0}^{T-2} \varrho^{2 j}\right)^{2} \leq C$

which suffices for the required uniform $L_{4}$-boundedness. 
To check condition ii), it suffices to show that

$$
\frac{1}{T^{1+\eta}} \sum_{t=2}^{T} \tilde{z}_{t-1}^{2} \varepsilon_{t}^{2}-s_{T}^{2} \stackrel{p}{\rightarrow} 0
$$

because $s_{T}^{2}$ is bounded and bounded away from zero (we learn from Lemma A.4 below that $s_{T}^{2} \rightarrow \frac{1}{2 a} \int_{0}^{1} \sigma_{\nu}^{2}(s) \sigma_{u}^{2}(s) \mathrm{d} s$, but the exact limit does not matter here). To prove (A.4), write

$$
\begin{aligned}
\sum_{t=2}^{T} \tilde{z}_{t-1}^{2} \varepsilon_{t}^{2}= & \sum_{t=2}^{T} \sum_{j=0}^{t-2} \sum_{k=0}^{t-2} \varrho^{j} \varrho^{k} \nu_{t-1-j} \nu_{t-1-k}\left(\varepsilon_{t}^{2}-\sigma_{\varepsilon, t}^{2}\right) \\
& +\sum_{t=2}^{T} \sum_{j=0}^{t-2} \sum_{k=0}^{t-2} \varrho^{j} \varrho^{k} \nu_{t-1-j} \nu_{t-1-k} \sigma_{\varepsilon, t}^{2} \\
= & : A_{T}+B_{T} .
\end{aligned}
$$

Note that $\sum_{j=0}^{t-2} \sum_{k=0}^{t-2} \varrho^{j} \varrho^{k} \nu_{t-1-j} \nu_{t-1-k}\left(\varepsilon_{t}^{2}-\sigma_{\varepsilon, t}^{2}\right)$ builds an md array and as such, is uncorrelated in $t$. Hence, showing $\frac{1}{T^{1+\eta}} A_{T}$ to vanish is not difficult, given that from the uncorrelatedness of the summands we can write that,

$$
\begin{aligned}
\operatorname{Var}\left(\frac{1}{T^{1+\eta}} A_{T}\right) & =\frac{1}{T^{2+2 \eta}} \sum_{t=2}^{T} \operatorname{Var}\left(\sum_{j=0}^{t-2} \sum_{k=0}^{t-2} \varrho^{j} \varrho^{k} \nu_{t-1-j} \nu_{t-1-k}\left(\varepsilon_{t}^{2}-\sigma_{\varepsilon, t}^{2}\right)\right) \\
& =\frac{1}{T^{2+2 \eta}} \sum_{t=2}^{T} \mathrm{E}\left(\left(\sum_{j=0}^{t-2} \sum_{k=0}^{t-2} \varrho^{j} \varrho^{k} \nu_{t-1-j} \nu_{t-1-k}\right)\right)^{2} \mathrm{E}\left(\left(\varepsilon_{t}^{2}-\sigma_{\varepsilon, t}^{2}\right)^{2}\right) .
\end{aligned}
$$

Now, $\varepsilon_{t}$ is uniformly $L_{4}$-bounded and

$$
\begin{aligned}
& \mathrm{E}\left(\left(\sum_{j=0}^{t-2} \sum_{k=0}^{t-2} \varrho^{j} \varrho^{k} \nu_{t-1-j} \nu_{t-1-k}\right)^{2}\right)= \\
& \quad \sum_{j=0}^{t-2} \sum_{k=0}^{t-2} \sum_{l=0}^{t-2} \sum_{m=0}^{t-2} \varrho^{j} \varrho^{k} \varrho^{l} \varrho^{m} \mathrm{E}\left(\nu_{t-1-j} \nu_{t-1-k} \nu_{t-1-l} \nu_{t-1-m}\right)
\end{aligned}
$$

where the expectation on the r.h.s. is, as before, uniformly bounded and nonzero only if the indices are pairwise equal. Hence,

$$
0 \leq\left(\left(\sum_{j=0}^{t-2} \sum_{k=0}^{t-2} \varrho^{j} \varrho^{k} \nu_{t-1-j} \nu_{t-1-k}\right)^{2}\right) \leq C \sum_{j=0}^{t-2} \sum_{k=0}^{t-2} \varrho^{2 j} \varrho^{2 k} \leq C T^{2 \eta}
$$


leading to $\operatorname{Var}\left(\frac{1}{T^{1+\eta}} A_{T}\right) \rightarrow 0$ and thus $A_{T}=o_{p}\left(T^{1+\eta}\right)$.

Regarding $B_{T}$, note that,

$$
\begin{aligned}
& B_{T}=T^{1+\eta} s_{T}^{2}+\sum_{t=2}^{T} \sum_{j=0}^{t-2} \varrho^{2 j}\left(\nu_{t-1-j}^{2}-\sigma_{\nu, t-1-j}^{2}\right) \sigma_{\varepsilon, t}^{2}
\end{aligned}
$$

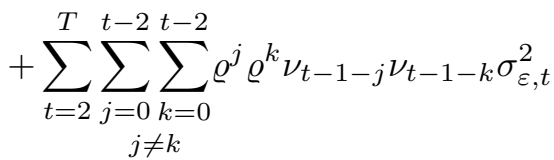

$$
\begin{aligned}
& =T^{1+\eta} s_{T}^{2}+B_{T 1}+B_{T 2} .
\end{aligned}
$$

For $B_{T 1}$ we have from the serial independence and $L_{4}$-boundedness of $\nu_{t}$ that

$$
\begin{aligned}
\left(\left(\sum_{j=0}^{t-2} \varrho^{2 j}\left(\nu_{t-1-j}^{2}-\sigma_{\nu, t-1-j}^{2}\right) \sigma_{\varepsilon, t}^{2}\right)^{2}\right) & =\sigma_{\varepsilon, t}^{4} \sum_{j=0}^{t-2} \varrho^{4 j}\left(\left(\nu_{t-1-j}^{2}-\sigma_{\nu, t-1-j}^{2}\right)^{2}\right) \\
& \leq C T^{\eta}
\end{aligned}
$$

and thus $\mathrm{E}\left(\left|\sum_{j=0}^{t-2} \varrho^{2 j}\left(\nu_{t-1-j}^{2}-\sigma_{\nu, t-1-j}^{2}\right) \sigma_{\varepsilon, t}^{2}\right|\right) \leq C T^{\eta / 2}$. Hence,

$$
\mathrm{E}\left(\left|\frac{1}{T^{1+\eta}} B_{T 1}\right|\right) \leq \frac{C}{T^{1+\eta}} \sum_{t=2}^{T} T^{\eta / 2} \rightarrow 0
$$

and Markov's inequality indicates that $B_{T 1}=o_{p}\left(T^{1+\eta}\right)$.

For $B_{T 2}$ we proceed similarly,

$$
\begin{aligned}
& \mathrm{E}\left(\left(\sum_{t=2}^{T} \sum_{j=0}^{t-2} \sum_{\substack{k=0 \\
j \neq k}}^{t-2} \varrho^{j} \varrho^{k} \nu_{t-1-j} \nu_{t-1-k} \sigma_{\varepsilon, t}^{2}\right)^{2}\right) \\
& =\sum_{t=2}^{T} \sum_{s=2}^{T} \sum_{j=0}^{t-2} \sum_{\substack{k=0 \\
j \neq k}}^{t-2} \sum_{\substack{s=0 \\
l \neq m}} \sum_{\substack{m=0 \\
l \neq m}}^{s-2} \varrho^{j} \varrho^{k} \varrho^{l} \varrho^{m} \sigma_{\varepsilon, t}^{2} \sigma_{\varepsilon, s}^{2} \mathrm{E}\left(\nu_{t-1-j} \nu_{t-1-k} \nu_{s-1-l} \nu_{s-1-m}\right),
\end{aligned}
$$

where the expectations on the r.h.s. are nonzero if $t-j=s-l$ and $t-k=s-m$ or if $t-j=s-m$ and $t-k=s-l$ (with $t-j=t-k$ and $s-l=s-m$ being excluded by the requirement that $j \neq k$ and $l \neq m)$. Note that, for any 
$t, s, j, k, l, m$ with $j \neq k$ and $l \neq m$,

$$
\sigma_{\varepsilon, t}^{2} \sigma_{\varepsilon, s}^{2}\left(\nu_{t-1-j} \nu_{t-1-k} \nu_{s-1-l} \nu_{s-1-m}\right) \leq\left(\max _{t} \sigma_{\varepsilon, t}^{2}\right)^{2}\left(\max _{t} \sigma_{\nu, t}^{2}\right)^{2} \leq C .
$$

Let us now focus on the terms for which $t-s=j-l=k-m$. Thus, for $t=s$, $t=2, \ldots, T$, we obtain

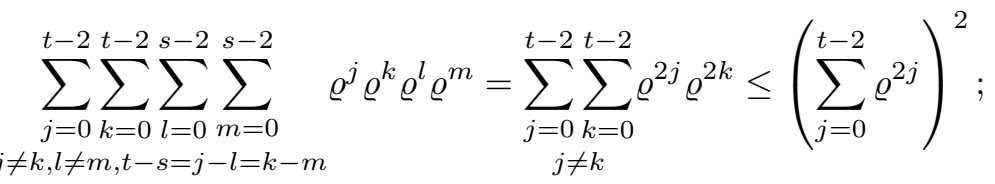

and for $s=t-1, t=3, \ldots, T$, we have analogously that,

$$
\sum_{\substack{j=0 \\ j \neq k, l \neq m, t-s=j-l=k-m}}^{t-2} \sum_{k=0}^{t-2} \sum_{m=0}^{s-2} \varrho^{j} \varrho^{k} \varrho^{l} \varrho^{m} \leq \varrho^{2}\left(\sum_{j=0}^{t-3} \varrho^{2 j}\right)^{2}
$$

while, for $s=t+1, t=2, \ldots, T-1$ (or equivalently $t=s-1, s=3, \ldots, T$ ), it follows that,

$$
\sum_{\substack{j=0 \\ j \neq k, l \neq m, t-s=j-l=k-m}}^{t-2} \sum_{\substack{t=0 \\ l-2}}^{s-2} \sum_{m=0}^{s-2} \varrho^{j} \varrho^{l} \varrho^{m} \leq \varrho^{2}\left(\sum_{l=0}^{s-3} \varrho^{2 l}\right)^{2} .
$$

Repeating the discussion for $s=t \pm r$ for $r=2, \ldots, T-2$, we have

$$
\sum_{\substack{j=0 \\ \neq k, l \neq m, t-s=j}}^{t-2} \sum_{\substack{l=0 \\ \text { t-2 }}}^{s-2} \sum_{m=0-m}^{s-2} \varrho^{j} \varrho^{k} \varrho^{l} \varrho^{m} \leq 2 \varrho^{2 r}\left(\sum_{j=0}^{t-r-2} \varrho^{2 j}\right)^{2}
$$

leading to

$$
\sum_{t=2}^{T} \sum_{s=2}^{T} \sum_{\substack{j=0 \\ j \neq k, l \neq m, t-s=j-l=k-m}}^{t-2} \sum_{k=0}^{t-2} \sum_{l=0}^{s-2} \sum_{m=0}^{s-2} \varrho^{j} \varrho^{k} \varrho^{l} \varrho^{m} \leq \sum_{t=2}^{T}\left(\sum_{j=0}^{t-2} \varrho^{2 j}\right)^{2}+2 \sum_{r=1}^{T-2} \varrho^{2 r} \sum_{t=2+r}^{T}\left(\sum_{j=0}^{t-r-2} \varrho^{2 j}\right)^{2} .
$$

The same holds when imposing $t-s=j-m=k-l$, such that, with $\sum_{j=0}^{t-r-2} \varrho^{2 j} \leq \sum_{j=0}^{T-1} \varrho^{2 j}$ and $\sum_{t=2+r}^{T} C \leq C T$, thus, we ultimately have

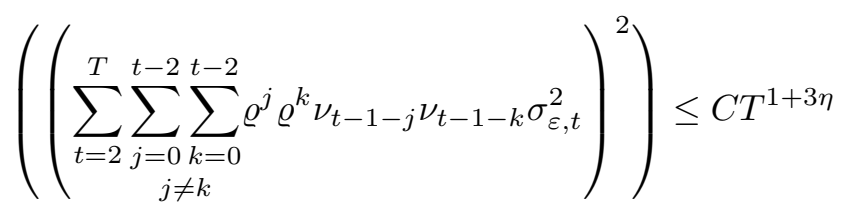


and consequently $B_{T 2}=o_{p}\left(T^{1+\eta}\right)$ when $\eta<1$, as required to complete the proof.

Lemma A.3. Under the assumptions of Theorem 2, it follows, as $T \rightarrow \infty$, that i) $\frac{\sum_{t=2}^{T} z_{t-1} \varepsilon_{t}}{\sqrt{\sum_{t=2}^{T} z_{t-1}^{2} \varepsilon_{t}^{2}}} \stackrel{d}{\rightarrow} \mathcal{N}(0,1)$; and ii) $\frac{\sum_{t=2}^{T} z_{t-1} u_{t}}{\sqrt{\sum_{t=2}^{T} z_{t-1}^{2} u_{t}^{2}}} \stackrel{d}{\rightarrow} \mathcal{N}(0,1)$.

Lemma A.3 suggests the use of White standard errors in the heteroskedastic near-integrated case, W.s.e $:=\frac{\sqrt{\sum_{t=2}^{T} z_{t-1}^{2} \hat{\varepsilon}_{t}^{2}}}{\sum_{t=2}^{T} z_{t-1}^{2}}$ with $\hat{\varepsilon}_{t}$ the OLS residuals guaranteeing $\sup _{2 \leq t \leq T}\left|\hat{\varepsilon}_{t}-\varepsilon_{t}\right| \stackrel{p}{\rightarrow} 0$ both in cases with and without intercept, and also better finite-sample behavior; see Kostakis et al. (2015). For the stable case, White standard errors are "mandatory" under time heteroskedasticity (Phillips and $\mathrm{Xu}$ 2006).

\section{Proof of LemmaA.3}

We first resort to the Phillips-Solo decomposition of $v_{t}$ and write $v_{t}=$ $\omega \nu_{t}+\Delta \tilde{v}_{t}$ where $\tilde{v}_{t}$ is a linear process in $\nu_{t}$ with exponentially decaying coefficients. Let also $\bar{z}_{t}:=(1-\varrho L)_{+}^{-1} v_{t}$. Thus, denoting $\tilde{z}_{t}=\sum_{j=0}^{t-1} \varrho^{j} \nu_{t-j}$ like in Lemma A.2, it follows that,

$$
\begin{aligned}
\bar{z}_{t} & =\omega \sum_{j=0}^{t-1} \varrho^{j} \nu_{t-j}+\left(\tilde{v}_{t}+(\varrho-1) \sum_{j=1}^{t-1} \varrho^{j-1} \tilde{v}_{t-j}-\varrho^{t-1} \tilde{v}_{1}\right) \\
& =\omega \tilde{z}_{t}+d_{t},
\end{aligned}
$$

and it can then easily be shown that $\operatorname{Var}\left(\sum_{j=1}^{t-1} \varrho^{j-1} \tilde{v}_{t-j}\right) \leq C T^{\eta}$ such that $d_{t}$ is uniformly $L_{2}$-bounded given that $\varrho-1=-a T^{-\eta}$. Similarly, $T^{-\eta / 2} \tilde{z}_{t}$ is uniformly $L_{2}$-bounded itself. We now show that

$$
\frac{1}{T^{1+\eta}} \sum_{t=2}^{T} \bar{z}_{t-1}^{2} \varepsilon_{t}^{2}=\frac{\omega^{2}}{T^{1+\eta}} \sum_{t=2}^{T} \tilde{z}_{t-1}^{2} \varepsilon_{t}^{2}+o_{p}(1)
$$

and

$$
\frac{1}{T^{1 / 2+\eta / 2}} \sum_{t=2}^{T} \bar{z}_{t-1} \varepsilon_{t}=\frac{\omega}{T^{1 / 2+\eta / 2}} \sum_{t=2}^{T} \tilde{z}_{t-1} \varepsilon_{t}+o_{p}(1) .
$$

Let us consider first (A.5). Note that,

$$
\frac{1}{T^{1+\eta}} \sum_{t=2}^{T} \bar{z}_{t-1}^{2} \varepsilon_{t}^{2}=\frac{\omega^{2}}{T^{1+\eta}} \sum_{t=2}^{T} \tilde{z}_{t-1}^{2} \varepsilon_{t}^{2}+\frac{2 \omega}{T^{1+\eta}} \sum_{t=2}^{T} \tilde{z}_{t-1} d_{t-1} \varepsilon_{t}^{2}+\frac{1}{T^{1+\eta}} \sum_{t=2}^{T} d_{t-1}^{2} \varepsilon_{t}^{2} .
$$


Since,

$$
\mathrm{E}\left(\left|d_{t-1}^{2} \varepsilon_{t}^{2}\right|\right)=\mathrm{E}\left(d_{t-1}^{2}\right) \mathrm{E}\left(\varepsilon_{t}^{2}\right)
$$

and

$$
\mathrm{E}\left(\left|\tilde{z}_{t-1} d_{t-1} \varepsilon_{t}^{2}\right|\right) \leq \sqrt{\mathrm{E}\left(\tilde{z}_{t-1}^{2}\right) \mathrm{E}\left(d_{t-1}^{2}\right)} \mathrm{E}\left(\varepsilon_{t}^{2}\right)
$$

due to the independence of $\varepsilon_{t}$ and $d_{t-1}$ and of $\varepsilon_{t}$ and $z_{t-1}$. With $\mathrm{E}\left(d_{t-1}^{2}\right), \mathrm{E}\left(\varepsilon_{t}^{2}\right)$ and $T^{-\eta} \mathrm{E}\left(\tilde{z}_{t-1}^{2}\right)$ being uniformly bounded, (A.5) then follows. To establish (A.6), write

$$
\frac{1}{T^{1 / 2+\eta / 2}} \sum_{t=2}^{T} \bar{z}_{t-1} \varepsilon_{t}=\frac{\omega}{T^{1 / 2+\eta / 2}} \sum_{t=2}^{T} \tilde{z}_{t-1} \varepsilon_{t}+\frac{1}{T^{1 / 2+\eta / 2}} \sum_{t=2}^{T} d_{t-1} \varepsilon_{t}
$$

and note that $d_{t-1} \varepsilon_{t}$ has the md property. Hence, $\sum_{t=2}^{T} d_{t-1} \varepsilon_{t}=O_{p}\left(T^{1 / 2}\right)$ due to the uniform $L_{2}$-boundedness and independence of $\varepsilon_{t}$ and $d_{t-1}$. Thus, from (A.5) and (A.6) we obtain that

$$
\frac{\sum_{t=2}^{T} \bar{z}_{t-1} \varepsilon_{t}}{\sqrt{\sum_{t=2}^{T} \bar{z}_{t-1}^{2} \varepsilon_{t}^{2}}}-\frac{\sum_{t=2}^{T} \tilde{z}_{t-1} \varepsilon_{t}}{\sqrt{\sum_{t=2}^{T} \tilde{z}_{t-1}^{2} \varepsilon_{t}^{2}}} \stackrel{p}{\rightarrow} 0
$$

In a second step we use the same reasoning to show that

$$
\frac{\sum_{t=2}^{T} \bar{z}_{t-1} \varepsilon_{t}}{\sqrt{\sum_{t=2}^{T} \bar{z}_{t-1}^{2} \varepsilon_{t}^{2}}}-\frac{\sum_{t=2}^{T} z_{t-1} \varepsilon_{t}}{\sqrt{\sum_{t=2}^{T} z_{t-1}^{2} \varepsilon_{t}^{2}}} \stackrel{p}{\rightarrow} 0
$$

Write to this end $z_{t}:=\bar{z}_{t}+r_{t}$ where $r_{t}:=-(1-\varrho L)_{+}^{-1} \frac{c_{t}}{T} x_{t-1}$ with

$\operatorname{Var}\left(\frac{1}{\sqrt{T}} x_{t}\right)=\frac{1}{T} \sum_{j=1}^{t} \sum_{k=1}^{t}\left(1-\frac{c_{t-j}}{T}\right)^{j}\left(1-\frac{c_{t-k}}{T}\right)^{k}\left(v_{t-j} v_{t-k}\right) \leq \frac{1}{T} \sum_{j=1}^{t} \sum_{k=1}^{t}\left|\left(v_{t-j} v_{t-k}\right)\right|$.

Given the uniform $L_{2}$-boundedness of the innovations $\nu_{t}$ and the exponential decay of the Wold coefficients of $v_{t}, \mathrm{E}\left|\left(v_{t-j} v_{t-k}\right)\right| \leq C e^{|j-k|} \forall t$ and $\frac{1}{\sqrt{T}} x_{t}$ is easily shown to be uniformly $L_{2}$-bounded.

The key in establishing (A.8) is to note that $r_{t-1}$ is independent of $\varepsilon_{t}$ and uniformly $L_{2}$-bounded, and that $T^{-\eta} \mathrm{E}\left(z_{t-1}^{2}\right)$ is uniformly bounded too whenever $T^{-\eta} \mathrm{E}\left(\bar{z}_{t-1}^{2}\right)$ and $\mathrm{E}\left(r_{t}^{2}\right)$ are. The arguments employed to show (A.7) thus apply for $z_{t}$ and $\bar{z}_{t}$ as well, and (A.8) holds.

Summing up, $\frac{\sum_{t=2}^{T} z_{t-1} \varepsilon_{t}}{\sqrt{\sum_{t=2}^{T} z_{t-1}^{2} \varepsilon_{t}^{2}}}$ and $\frac{\sum_{t=2}^{T} \tilde{z}_{t-1} \varepsilon_{t}}{\sqrt{\sum_{t=2}^{T} \tilde{z}_{t-1}^{2} \varepsilon_{t}^{2}}}$ are asymptotically equivalent and the result follows from Lemma A.2. 
The proof of the result in ii) follows along the same lines and we omit the details

LEMmA A.4. Under the assumptions of Theorem 2, it holds, as $T \rightarrow \infty$, that

1. $\frac{1}{T^{1+\eta}} \sum_{t=p+1}^{T} z_{t-1}^{2} \varepsilon_{t}^{2} \stackrel{p}{\rightarrow} \frac{\omega^{2}}{2 a} \int_{0}^{1} \sigma_{\nu}^{2}(s) \sigma_{\varepsilon}^{2}(s) \mathrm{d} s$;

2. $\frac{1}{T^{1+\eta}} \sum_{t=p+1}^{T} z_{t-1}^{2} u_{t}^{2} \stackrel{p}{\rightarrow} \frac{\omega^{2}}{2 a} \int_{0}^{1} \sigma_{\nu}^{2}(s) \sigma_{u}^{2}(s) \mathrm{d} s$ where $\sigma_{u}^{2}(s)=\sigma_{\varepsilon}^{2}(s)+$ $\gamma^{2} \sigma_{\nu}^{2}(s)$

3. $\frac{1}{T^{1+\eta}} \sum_{t=p+1}^{T} z_{t-1} x_{t-1} \Rightarrow \frac{\omega^{2}}{a}\left(X^{2}(1)-\int_{0}^{1} X(s) \mathrm{d} X(s)\right)$

where $X(r)$ is an Ornstein-Uhlenbeck process as defined in (14).

\section{Proof of Lemma A.4}

1. To obtain the limit of $\frac{1}{T^{1+\eta}} \sum_{t=p+1}^{T} z_{t-1}^{2} \varepsilon_{t}^{2}$, we use from the proof of Lemma A.3 (see (A.2)) the fact that

$$
\frac{1}{T^{1+\eta}} \sum_{t=p+1}^{T} z_{t-1}^{2} \varepsilon_{t}^{2}=\omega^{2} \frac{1}{T^{1+\eta}} \sum_{t=2}^{T} \sum_{j=0}^{t-2} \varrho^{2 j} \sigma_{\nu, t-1-j}^{2} \sigma_{\varepsilon, t}^{2}+o_{p}(1) .
$$

The Lipschitz property implies that $\left|\sigma_{\nu, t-1-j}^{2}-\sigma_{\nu, t}^{2}\right| \leq C \frac{j}{T}$ such that $0 \leq \frac{1}{T^{1+\eta}}\left|\sum_{t=2}^{T} \sum_{j=0}^{t-2} \varrho^{2 j} \sigma_{\nu, t-1-j}^{2} \sigma_{\varepsilon, t}^{2}-\sum_{t=2}^{T} \sigma_{\nu, t}^{2} \sigma_{\varepsilon, t}^{2} \sum_{j=0}^{t-2} \varrho^{2 j}\right| \leq C \frac{1}{T^{2+\eta}} \sum_{t=2}^{T} \sum_{j=0}^{t-2} j \varrho^{2 j}$

On the r.h.s. we have immediately, as $\mathrm{T} \rightarrow \infty$, that

$$
\frac{1}{T^{2+\eta}} \sum_{t=2}^{T} \sum_{j=0}^{t-2} j \varrho^{2 j} \rightarrow 0
$$

given that $\sum_{j=0}^{t-2} j \varrho^{2 j}=\frac{t \varrho^{2(t-3)}(\varrho-1)-\left(\varrho^{2(t-2)}-1\right)}{\left(\varrho^{2}-1\right)^{2}}$, where $\left|\frac{t \varrho^{2(t-3)}(\varrho-1)}{\left(\varrho^{2}-1\right)^{2}}\right| \leq$ $C T^{1+\eta} \varrho^{2(t-3)}$ and $\left|\frac{\varrho^{2(t-2)}-1}{\left(\varrho^{2}-1\right)^{2}}\right| \leq C T^{2 \eta}$. We also observe that,

$$
\begin{aligned}
\frac{1}{T^{1+\eta}} \sum_{t=2}^{T} \sigma_{\nu, t}^{2} \sigma_{\varepsilon, t}^{2} \sum_{j=0}^{t-2} \varrho^{2 j}= & \frac{1}{T^{1+\eta}} \sum_{t=2}^{T} \sigma_{\nu, t}^{2} \sigma_{\varepsilon, t}^{2} \frac{T^{\eta}}{a}\left(\frac{1-\varrho^{2(t-1)}}{1+\varrho}\right) \\
= & \frac{1}{T^{1+\eta}} \sum_{t=2}^{T} \sigma_{\nu, t}^{2} \sigma_{\varepsilon, t}^{2} \frac{T^{\eta}}{a(1+\varrho)}- \\
& -\frac{1}{T^{1+\eta}} \sum_{t=2}^{T} \sigma_{\nu, t}^{2} \sigma_{\varepsilon, t}^{2} \frac{T^{\eta}}{a}\left(\frac{\varrho^{2(t-1)}}{1+\varrho}\right)
\end{aligned}
$$


The first summand on the r.h.s. is easily seen to converge to $\frac{1}{2 a} \int_{0}^{1} \sigma_{\nu}^{2}(s) \sigma_{\varepsilon}^{2}(s) \mathrm{d} s$, while, for the second, we have

$$
\frac{1}{T^{1+\eta}} \sum_{t=2}^{T} \sigma_{\nu, t}^{2} \sigma_{\varepsilon, t}^{2} \frac{T^{\eta}}{a}\left(\frac{\varrho^{2(t-1)}}{1+\varrho}\right) \leq \frac{C}{a T} \sum_{t=2}^{T} \varrho^{2(t-1)}=O\left(T^{\eta-1}\right)=o(1)
$$

as required to complete the proof.

2. The proof of 2 is analogous to the proof of 1 and is therefore omitted.

3. Let $S_{t}:=\sum_{j=2}^{t} z_{t}$. We first follow Breitung and Demetrescu (2015, Proof of Corollary 1.2) and show that

$$
\frac{1}{T^{1 / 2+\eta}} S_{t}=\frac{1}{a \sqrt{T}} x_{t}+o_{p}(1)
$$

where the $o_{p}(1)$ term is uniform. The arguments are essentially the same as there; the only difference is having to show that $\mathrm{E}\left(\left|x_{t}-x_{t-j}\right|\right) \leq C \sqrt{j}$ for all $t$ and $j$, which is obvious in their i.i.d. setup, but marginally more difficult here. To this end, recall that $\Delta x_{t}:=v_{t}-\frac{c_{t-1}}{T} x_{t-1}$ and use Liapunov's and Minkowski's inequalities to conclude that,

$$
\begin{aligned}
\mathrm{E}\left(\left|x_{t}-x_{t-j}\right|\right) & \leq \sqrt{\mathrm{E}\left(\left(x_{t}-x_{t-j}\right)^{2}\right)}=\sqrt{\mathrm{E}\left(\left(\sum_{k=0}^{j-1} v_{t-j}-\frac{1}{T} \sum_{k=0}^{j-1} c_{t-k-1} x_{t-k-1}\right)^{2}\right)} \\
& \leq \sqrt{\mathrm{E}\left(\left(\sum_{k=0}^{j-1} v_{t-j}\right)^{2}\right)}+\frac{1}{\sqrt{T}} \sum_{k=0}^{j-1}\left|c_{t-k-1}\right| \sqrt{\mathrm{E}\left(\left(\frac{x_{t-k-1}}{\sqrt{T}}\right)^{2}\right)}
\end{aligned}
$$

and therefore using the uniform boundedness of the variance of $\frac{x_{t-k-1}}{\sqrt{T}}$, it follows indeed that $\left(\left|x_{t}-x_{t-j}\right|\right) \leq C \sqrt{j}$ as required.

We then follow Breitung and Demetrescu (2015, Proof of Theorem 2) and obtain via partial summation that,

$$
\begin{aligned}
\frac{1}{T^{1+\eta}} \sum_{t=p+1}^{T} z_{t-1} x_{t-1} & =\frac{1}{T^{1+\eta}} \sum_{t=p+1}^{T}\left(S_{t-1}-S_{t-2}\right) x_{t-1} \\
& =\frac{1}{T^{1+\eta}}\left(S_{T-1} x_{T-1}-S_{p-1} x_{p}\right)-\frac{1}{T^{1+\eta}} \sum_{t=p+1}^{T} S_{t-2} \Delta x_{t-1} .
\end{aligned}
$$


Now, since $S_{p-1} x_{p}=O_{p}(1)$ it is negligible in the limit; furthermore note that,

$$
\frac{1}{T^{1+\eta}} \sum_{t=p+1}^{T} S_{t-2} \Delta x_{t-1}=\frac{1}{T^{1+\eta}} \sum_{t=p+1}^{T} S_{t-2} v_{t-1}-\frac{1}{T^{2+\eta}} \sum_{t=p+1}^{T} c_{t-2} S_{t-2} x_{t-2} .
$$

For the first summand on the r.h.s., we have using the Phillips-Solo device for the AR process $v_{t-1}$ that,

$$
\begin{aligned}
\frac{1}{T^{1+\eta}} \sum_{t=p+1}^{T} S_{t-2} v_{t-1} & =\frac{\omega}{T^{1+\eta}} \sum_{t=p+1}^{T} S_{t-2} \nu_{t-1}+\frac{1}{T^{1+\eta}} \sum_{t=p+1}^{T} S_{t-2} \Delta \tilde{v}_{t-1} \\
& =: A_{T}+B_{T},
\end{aligned}
$$

where $\tilde{v}_{t}$ is a linear process with exponentially decaying coefficients.

Since $\nu_{t-1}$ is independent of $S_{t-2}$ and the conditions of Hansen (1992) are fulfilled, we have that,

$$
A_{T} \Rightarrow \frac{\omega^{2}}{a} \int_{0}^{1} X(s) \mathrm{d} M(s)
$$

Using the partial summation formula on $B_{T}$, it follows that,

$$
B_{T}=\frac{1}{T^{1+\eta}}\left(\tilde{v}_{T-1} S_{T-2}-\tilde{v}_{p-1} S_{p-1}\right)-\frac{1}{T^{1+\eta}} \sum_{t=p+1}^{T} \tilde{v}_{t-2} \Delta S_{t-2} .
$$

Since $\sup _{1 \leq t \leq T}\left|S_{t}\right|=T^{\eta} \sup _{1 \leq t \leq T}\left|x_{t}\right|+o_{p}\left(T^{1 / 2+\eta}\right)=O_{p}\left(T^{1 / 2+\eta}\right)$ and $\tilde{v}_{p-1} S_{p-1}=O_{p}(1)$, it follows that the first summand on the r.h.s. of the above equation is negligible; for the second, we have

$$
\frac{1}{T^{1+\eta}} \sum_{t=p+1}^{T} \tilde{v}_{t-2} \Delta S_{t-2}=\frac{1}{T^{1+\eta}} \sum_{t=p+1}^{T} \tilde{v}_{t-2} z_{t-2} .
$$

Clearly, $\tilde{v}_{t-2}$ is uniformly $L_{2}$-bounded, and it is easily shown that $T^{-\eta / 2} z_{t}$ is uniformly $L_{2}$-bounded as well. Then, the Cauchy-Schwarz inequality indicates that $\mathrm{E}\left(\left|\tilde{v}_{t-2} z_{t-2}\right|\right)<C T^{\eta / 2}$ such that

$$
\mathrm{E}\left(\left|\frac{1}{T^{1+\eta}} \sum_{t=p+1}^{T} \tilde{v}_{t-2} \Delta S_{t-2}\right|\right) \leq C T^{-\eta / 2}
$$

and $\frac{1}{T^{1+\eta}} \sum_{t=p+1}^{T} \tilde{v}_{t-2} \Delta S_{t-2}$ vanishes in probability. 
Hence

$$
\frac{1}{T^{1+\eta}} \sum_{t=p+1}^{T} z_{t-1} x_{t-1}=\frac{1}{a} \frac{x_{T-1}^{2}}{T}-\frac{1}{a}\left(\frac{a \omega}{T^{1+\eta}} \sum_{t=p+1}^{T} S_{t-2} \nu_{t-1}-\frac{1}{T^{2}} \sum_{t=p+1}^{T} c_{t-2} x_{t-2}^{2}\right)+o_{p}(1) .
$$

Using the weak convergence of $S_{t}$ and $x_{t}$ we obtain

$$
\begin{aligned}
\frac{1}{T^{1+\eta}} \sum_{t=p+1}^{T} z_{t-1} x_{t-1} & \Rightarrow \frac{\omega^{2}}{a} X^{2}(1)-\frac{\omega^{2}}{a}\left(\int_{0}^{1} X(s) \mathrm{d} M(s)-\int_{0}^{1} c(s) X^{2}(s) \mathrm{d} s\right) \\
& \equiv \frac{\omega^{2}}{a}\left(X^{2}(1)-\int_{0}^{1} X(s) \mathrm{d} X(s)\right) .
\end{aligned}
$$

Note that, interestingly, $\frac{1}{T^{1+\eta}} \sum_{t=p+1}^{T} S_{t-2} v_{t-1}$ converges to an Itô-type integral without bias term, unlike $\frac{1}{T^{1}} \sum_{t=p+1}^{T} x_{t-2} v_{t-1}$ under serial correlation. This is because $S_{t}$ and $x_{t}$ require different normalizations, which is essentially the expression of the same mechanism ensuring mixed Gaussianity of the unadjusted IVX estimator.

\section{Proof of Theorem 1}

Consider

$$
\tilde{\beta}^{i v x}:=\frac{\sum_{t=p+1}^{T} z_{t-1} \tilde{y}_{t}}{\sum_{t=p+1}^{T} z_{t-1} x_{t-1}}
$$

Since $\tilde{y}_{t}:=y_{t}-\hat{\gamma} \hat{\nu}_{t}=\beta x_{t-1}+\gamma \nu_{t}-\hat{\gamma} \hat{\nu}_{t}+\varepsilon_{t}$ it follows that we can express $\tilde{\beta}^{i v x}$ as,

$$
\tilde{\beta}^{i v x}:=\frac{\sum_{t=p+1}^{T} z_{t-1} \tilde{y}_{t}}{\sum_{t=p+1}^{T} z_{t-1} x_{t-1}}=\beta+\frac{\sum_{t=p+1}^{T} z_{t-1}\left(\gamma \nu_{t}-\hat{\gamma} \hat{\nu}_{t}+\varepsilon_{t}\right)}{\sum_{t=p+1}^{T} z_{t-1} x_{t-1}}
$$

Write for the stable autoregression case

$$
\hat{\nu}_{t}:=\nu_{t}-(\hat{\mathbf{a}}-\mathbf{a})^{\prime} \mathbf{x}_{t-p}
$$

with $\mathbf{x}_{t-p}$ stacking the $p$ lags of $x_{t}$ and a the corresponding coefficients (of $(1-\rho L) A(L))$, i.e. the pure autoregressive representation of $x_{t}$. 
Then, analyze

$$
\begin{aligned}
z_{t-1} & =\sum_{j=0}^{t-3} \varrho^{j} \Delta x_{t-1-j} \\
& =x_{t-1}-\varrho^{t-3} x_{1}+(\varrho-1) \sum_{j=0}^{t-4} \varrho^{j} x_{t-2-j} .
\end{aligned}
$$

We have that

$$
(\varrho-1) \sum_{j=0}^{t-4} \varrho^{j} x_{t-2-j}=-\frac{a}{T^{\eta}} \sum_{j=0}^{t-4} \varrho^{j} x_{t-2-j}=-\frac{a}{T^{\eta}} d_{t-2}
$$

where $d_{t-2}$ is here, with $x_{t}$ a stable autoregression, a mildly integrated process which is known to be $O_{p}\left(T^{\eta / 2}\right)$. Furthermore, $\varrho^{t-3} \rightarrow 0$ when $t$ goes to infinity at suitable rates; in the derivations below, the effect will be quantified precisely whenever needed, but it is important to keep in mind that $z_{t-1} \approx x_{t-1}$ which is a stable autoregression.

We thus have for the numerator of $\tilde{\beta}^{i v x}-\beta$ in (A.10) that,

$$
\sum_{t=p+1}^{T} z_{t-1}\left(\varepsilon_{t}+\gamma \nu_{t}-\hat{\gamma} \hat{\nu}_{t}\right)=\sum_{t=p+1}^{T} z_{t-1} \varepsilon_{t}-\gamma \sum_{t=p+1}^{T} z_{t-1}\left(\hat{\nu}_{t}-\nu_{t}\right)-(\hat{\gamma}-\gamma) \sum_{t=p+1}^{T} z_{t-1} \hat{\nu_{t}} .
$$

The first two summands in (A.11) deliver a normal distribution. This is because

$$
\begin{aligned}
\frac{1}{T^{1 / 2}} \sum_{t=p+1}^{T} z_{t-1} \varepsilon_{t} & =\frac{1}{T^{1 / 2}} \sum_{t=p+1}^{T} x_{t-1} \varepsilon_{t}-\frac{a}{T^{1 / 2+\eta}} \sum_{t=p+1}^{T} d_{t-2} \varepsilon_{t}+\frac{x_{1}}{T^{1 / 2}} \sum_{t=p+1}^{T} \varrho^{t-3} \varepsilon_{t} \\
& =\frac{1}{T^{1 / 2}} \sum_{t=p+1}^{T} x_{t-1} \varepsilon_{t}+o_{p}(1)
\end{aligned}
$$

with $\sum_{t=p+1}^{T} d_{t-2} \varepsilon_{t}=O_{p}\left(T^{1 / 2+\eta / 2}\right)$ given the results in the proofs of Lemmas A.2 and A.3, and $\sum_{t=p+1}^{T} \varrho^{t-3} \varepsilon_{t}=O_{p}\left(T^{\eta / 2}\right)$ given that $\operatorname{Var}\left(\sum_{t=p+1}^{T} \varrho^{t-3} \varepsilon_{t}\right)=O_{p}\left(\sum_{t=p+1}^{T} \varrho^{2 t}\right)=O_{p}\left(T^{\eta}\right)$. Furthermore,

$$
\frac{1}{T^{1 / 2}} \sum_{t=p+1}^{T} z_{t-1}\left(\hat{\nu}_{t}-\nu_{t}\right)=-\left(\frac{1}{T} \sum_{t=p+1}^{T} z_{t-1} \mathbf{x}_{t-p}^{\prime}\right) \sqrt{T}(\hat{\mathbf{a}}-\mathbf{a}),
$$

where the OLS autoregressive estimators,

$$
\sqrt{T}(\hat{\mathbf{a}}-\mathbf{a})=\left(\frac{1}{T} \sum_{t=p+1}^{T} \mathbf{x}_{t-p} \mathbf{x}_{t-p}^{\prime}\right)^{-1} \frac{1}{\sqrt{T}} \sum_{t=p+1}^{T} \mathbf{x}_{t-p} \nu_{t}
$$


following standard arguments can be shown to have a limiting multivariate normal distribution. We now show that $\frac{1}{T} \sum_{t=2}^{T} z_{t-1} \mathbf{x}_{t-p}$ does not converge to a vector of zeros, such that the limiting distribution of $\frac{1}{T^{1 / 2}} \sum_{t=p+1}^{T} z_{t-1}\left(\hat{\nu}_{t}-\nu_{t}\right)$ is driven by $\frac{1}{\sqrt{T}} \sum_{t=p+1}^{T} \mathbf{x}_{t-p} \nu_{t}$. Given that

$\frac{1}{T} \sum_{t=p+1}^{T} z_{t-1} \mathbf{x}_{t-p}=\frac{1}{T} \sum_{t=p+1}^{T} x_{t-1} \mathbf{x}_{t-p}-\frac{1}{T} \sum_{t=p+1}^{T} \varrho^{t-3} x_{1} \mathbf{x}_{t-p}-\frac{a}{T^{1+\eta}} \sum_{t=p+1}^{T} d_{t-2} \mathbf{x}_{t-p}$,

the first summand on the r.h.s. gives the desired limit (see Lemma A.1). The second is easily seen to vanish since $\left(x_{1} x_{t}\right)$ vanishes at exponential rate (in $\left.t\right)$. For the third, we show that $\sum_{t=p+1}^{T} d_{t-2} \mathbf{x}_{t-p}=O_{p}(T)$ as follows. By resorting to the Phillips-Solo device, it is tedious, yet straightforward to show that

$\frac{1}{T} \sum_{t=p+1}^{T} d_{t-2} \mathbf{x}_{t-p}=O_{p}\left(\frac{1}{T} \sum_{t=p+1}^{T} \tilde{d}_{t-2} \nu_{t-p}\right) \quad$ where $\quad \tilde{d}_{t-2}:=\sum_{j=0}^{t-3} \varrho^{j} \nu_{t-2-j}$.

Then,

$$
\frac{1}{T} \sum_{t=p+1}^{T} \tilde{d}_{t-2} \nu_{t-p}=\frac{1}{T} \sum_{t=p+1}^{T} \tilde{d}_{t-p-1} \nu_{t-p}+O_{p}(1)
$$

and the proofs of Lemmas A.2 and A.3 provide the arguments leading to $\frac{1}{T} \sum_{t=p+2}^{T} \tilde{d}_{t-p-1} \nu_{t-p}=O_{p}\left(\frac{T^{1 / 2+\eta / 2}}{T}\right)=O_{p}(1)$ as required.

The third summand in (A.11) is

$$
\begin{aligned}
\frac{\hat{\gamma}-\gamma}{\sqrt{T}} \sum_{t=p+1}^{T} z_{t-1} \hat{\nu}_{t} & =(\hat{\gamma}-\gamma)\left(\frac{1}{\sqrt{T}} \sum_{t=p+1}^{T} z_{t-1} \nu_{t}+\frac{1}{\sqrt{T}} \sum_{t=p+1}^{T} z_{t-1}\left(\hat{\nu}_{t}-\nu_{t}\right)\right) \\
& =o_{p}(1)
\end{aligned}
$$

since $\hat{\gamma}$ is easily shown to be consistent for $\gamma, \frac{1}{\sqrt{T}} \sum_{t=p+1}^{T} z_{t-1} \nu_{t}=O_{p}(1)$ like in the case of $\frac{1}{\sqrt{T}} \sum_{t=p+1}^{T} z_{t-1} \varepsilon_{t}$, and $\frac{1}{\sqrt{T}} \sum_{t=p+1}^{T} z_{t-1}\left(\hat{\nu}_{t}-\nu_{t}\right)=O_{p}(1)$ as above. 
Hence,

$$
\begin{aligned}
& \frac{1}{\sqrt{T}} \sum_{t=p+1}^{T} z_{t-1}\left(\varepsilon_{t}+\gamma \nu_{t}-\hat{\gamma} \hat{\nu}_{t}\right) \\
& =\frac{1}{\sqrt{T}} \sum_{t=p+1}^{T} z_{t-1} \varepsilon_{t}+\gamma\left(\frac{1}{T} \sum_{t=p+1}^{T} z_{t-1} \mathbf{x}_{t-p}^{\prime}\right)\left(\frac{1}{T} \sum_{t=p+1}^{T} \mathbf{x}_{t-p} \mathbf{x}_{t-p}^{\prime}\right)^{-1} \times \\
& \quad \times \frac{1}{\sqrt{T}} \sum_{t=p+1}^{T} \mathbf{x}_{t-p} \nu_{t}+o_{p}(1) .
\end{aligned}
$$

Furthermore, it is shown along the lines of the discussion of $T^{-1} \sum_{p+1}^{T} z_{t-1} \mathbf{x}_{t-p}$ that

$$
\frac{1}{\sqrt{T}} \sum_{t=p+1}^{T} z_{t-1} \varepsilon_{t}=\frac{1}{\sqrt{T}} \sum_{t=p+1}^{T} x_{t-1} \varepsilon_{t}+o_{p}(1) .
$$

for both $\frac{1}{\sqrt{T}} \sum_{t=p+1}^{T} z_{t-1} \varepsilon_{t}$ and $\frac{1}{\sqrt{T}} \sum_{t=p+1}^{T} \mathbf{x}_{t-p} \nu_{t}$, Theorem 24.3 in Davidson (1994) is easily checked to apply (see Lemma A.1 for the convergence of the sample covariance matrices); since $\mathbf{x}_{t-p} \nu_{t}$ and $z_{t-1} \varepsilon_{t}$ are orthogonal thanks to the uncorrelatedness of $\nu_{t}$ and $\varepsilon_{t}$, the term $\frac{1}{\sqrt{T}} \sum_{t=p+1}^{T} z_{t-1}\left(\varepsilon_{t}+\gamma \nu_{t}-\hat{\gamma} \hat{\nu}_{t}\right)$ is asymptotically normal with mean zero and asymptotic variance

$$
\alpha_{0} \int_{0}^{1} \sigma_{v}^{2}(s) \sigma_{\varepsilon}^{2}(s) \mathrm{d} s+\gamma^{2}\left(\alpha_{0} \ldots \alpha_{p-1}\right) \Omega^{-1}\left(\alpha_{0} \ldots \alpha_{p-1}\right)^{\prime} \int_{0}^{1} \sigma_{v}^{4}(s) \mathrm{d} s .
$$

Checking that

$$
\frac{1}{T} \sum_{t=p+1}^{T} z_{t-1}^{2} \hat{\varepsilon}_{t}^{2}+\frac{1}{T} \hat{\gamma}^{2} \hat{Q}_{T}
$$

estimates the above asymptotic variance consistently is straightforward and we omit the details.

\section{Proof of Theorem 2}

Standard OLS algebra shows that the residuals $\hat{\nu}_{t}$ are numerically the same as in the autoregressive representation of $x_{t}$ if resorting to the error-correction representation, which is more convenient with near-integration. We may thus write

$$
\hat{\nu}_{t}:=\nu_{t}-(\hat{\varphi}-\varphi) x_{t-1}-(\hat{\alpha}-\alpha)^{\prime} \Delta \mathbf{x}_{t-p+1}
$$


with $\Delta \mathbf{x}_{t-p+1}$ stacking the first $p-1$ lags of $\Delta x_{t}$ and $\varphi:=\frac{1}{\omega}(\rho-1)$ (the vector $\alpha$ depends on all autoregressive coefficients of $x_{t}$, but its exact value is irrelevant here).

We have the same representation as in (A.11), i.e.,

$$
\begin{aligned}
& \sum_{t=p+1}^{T} z_{t-1}\left(\varepsilon_{t}+\gamma \nu_{t}-\hat{\gamma} \hat{\nu}_{t}\right)=\sum_{t=p+1}^{T} z_{t-1} \varepsilon_{t}- \\
& -\gamma \sum_{t=p+1}^{T} z_{t-1}\left(\hat{\nu}_{t}-\nu_{t}\right)-(\hat{\gamma}-\gamma) \sum_{t=p+1}^{T} z_{t-1} \hat{\nu}_{t},
\end{aligned}
$$

yet $z_{t}$ is now a mildly integrated variable. Still, Lemmas A.3 and A.4 show that

$$
\frac{1}{T^{1 / 2+\eta / 2}} \sum_{t=p+1}^{T} z_{t-1} \varepsilon_{t}
$$

is asymptotically normal with variance $\omega^{2} \int_{0}^{1} \sigma_{v}^{2}(s) \sigma_{\varepsilon}^{2}(s) \mathrm{d} s$, whereas the remaining term can be re-written as

$$
\begin{gathered}
\frac{1}{T^{1 / 2+\eta / 2}} \sum_{t=p+1}^{T} z_{t-1}\left(\hat{\nu}_{t}-\nu_{t}\right)=-\frac{1}{T^{1 / 2+\eta / 2}} \sum_{t=p+1}^{T} z_{t-1} x_{t-1}(\hat{\varphi}-\varphi)- \\
-\frac{1}{T^{1 / 2+\eta / 2}} \sum_{t=p+1}^{T} z_{t-1} \Delta \mathbf{x}_{t-p+1}^{\prime}(\hat{\alpha}-\alpha) .
\end{gathered}
$$

In the limit, this vanishes because $(\hat{\varphi}-\varphi)$ is $O_{p}\left(T^{-1}\right)$ and $(\hat{\alpha}-\alpha)=$ $O_{p}\left(T^{-1 / 2}\right)$ as standard analysis of near-unit root autoregressions shows, while, at the same time,

$$
\sum_{t=p+1}^{T} z_{t-1} x_{t-1}=O_{p}\left(T^{1+\eta}\right)
$$

(see Lemma A.4.3) and we only need to show that

$$
\sum_{t=p+1}^{T} z_{t-1} \Delta \mathbf{x}_{t-p+1}^{\prime}=O_{p}(T) .
$$

This is known to be the case when $z_{t-1}$ is a near-integrated or stationary variable; we discuss here the case where $z_{t}$ is an IVX instrument. Examining 
$\sum_{t=p+2}^{T} z_{t-1} \Delta x_{t-1}$ as a representative for the whole vector,

$$
\frac{1}{T} \sum_{t=p+1}^{T} z_{t-1} \Delta x_{t-1}=\frac{1}{T} \sum_{t=p+1}^{T} z_{t-1} v_{t-1}+\frac{1}{T^{2}} \sum_{t=p+1}^{T} c_{t} z_{t-1} x_{t-2},
$$

it is easily shown that both $\frac{z_{t}}{\sqrt{T}}$ and $\frac{x_{t}}{\sqrt{T}}$ are uniformly $L_{2}$-bounded, hence $\mathrm{E}\left(\frac{1}{T^{2}} \sum_{t=p+1}^{T} c_{t} z_{t-1} x_{t-2}\right)=O(1)$. Moreover, $\frac{1}{T} \sum_{t=p+1}^{T} z_{t-1} v_{t-1}$ is itself $O_{p}(1)$, which can be shown along the lines of the discussion for $\frac{1}{T} \sum q_{t-2} \mathbf{x}_{t-p}$ in the proof of Theorem 1.

\section{Proof of Theorem 3}

Since the residual effect of $\varepsilon_{t}$ and $\nu_{t}$ is easily checked to be negligible, the correction $Q_{T}$ is negligible under the local alternative as well and we have for the residual-augmented IVX $t$-statistic that,

$$
\begin{aligned}
\tilde{t}_{\beta_{1}}^{i v x} & =\frac{\sum_{t=p+1}^{T} z_{t-1}\left(\varepsilon_{t}+\beta_{1} x_{t-1}\right)}{\sqrt{\sum_{t=p+1}^{T} z_{t-1}^{2} \varepsilon_{t}^{2}}}+o_{p}(1) \\
& =\frac{\sum_{t=p+1}^{T} z_{t-1} \varepsilon_{t}}{\sqrt{\sum_{t=p+1}^{T} z_{t-1}^{2} \varepsilon_{t}^{2}}}+b \frac{\frac{1}{T^{1+\eta}} \sum_{t=p+1}^{T} z_{t-1} x_{t-1}}{\sqrt{\frac{1}{T^{1+\eta}} \sum_{t=p+1}^{T} z_{t-1}^{2} \varepsilon_{t}^{2}}}+o_{p}(1) .
\end{aligned}
$$

The first summand on the r.h.s. converges to a standard normal distribution, $\mathcal{Z}$; note that $\mathcal{Z}$ would indeed be independent of the limit process of the regressor $x_{t}$ since $z_{t-1} \varepsilon_{t}$ and $\nu_{t}$ are orthogonal. Thus, the result follows with Lemma A.4, items 1 and 3 . 


\section{Appendix B: Tests for Persistence Change}

In this section, for completeness, we provide a brief overview of the persistence change tests of Harvey et al. (2006), which where used to evaluate whether the series under analysis had undergone some persistence change over time.

\section{B.1. The generic persistence change model}

We follow Harvey et al. (2006) and Busetti and Taylor (2004) and consider the following data generation process (DGP),

$$
\begin{aligned}
& x_{t}=d_{t}^{\prime} \beta+r_{t} \\
& r_{t}=\rho_{t} r_{t-1}+v_{t}
\end{aligned}
$$

where $r_{0}=0, d_{t}$ is a set of deterministic variables, such as a constant or, if necessary, a constant and time trend, $v_{t}$ is taken to satisfy Assumption 3 (together with 2), and $\rho_{t}$ obeys Assumption 4 in the most general case. For compatibility with the existing literature on testing for changes in persistence we shall assume the variance functions in Assumption 2 to be constant throughout.

Four relevant hypothesis can be considered:

1. $H_{1}: x_{t}$ is $I(1)$ (i.e. nonstationary) throughout the sample period. Harvey et al. (2006) set $\rho_{t}=1-\frac{c}{T}, c \geq 0$, so as to allow for unit root and near unit root behaviour.

2. $H_{01}: x_{t}$ is $I(0)$ changing to $I(1)$ (in other words, stationary changing to nonstationary) at time $\left[\tau^{*} T\right]$; that is $\rho_{t}=\rho, \rho<1$ for $t \leq\left[\tau^{*} T\right]$ and $\rho_{t}=1-\frac{c}{T}$ for $t>\left[\tau^{*} T\right]$. The change point proportion, $\tau^{*}$, is assumed to be an unknown point in $\Lambda=\left[\tau_{l}, \tau_{u}\right]$, an interval in $(0,1)$ which is symmetric around 0.5;

3. $H_{10}: x_{t}$ is $I(1)$ changing to $I(0)$ (i.e. nonstationary changing to stationary) at time $\left[\tau^{*} T\right]$;

4. $H_{0}: x_{t}$ is $I(0)$ (stationary) throughout the sample period.

\section{B.2. The ratio-based persistence change tests}

In the context of no breaks, Kim (2000), Kim et al. (2002) and Busetti and Taylor (2004) introduced tests for the constant $I(0)$ DGP $\left(H_{0}\right)$ against the 
$I(0)-I(1)$ change $\left(H_{01}\right)$ which are based on the ratio statistic,

$$
K_{[\tau T]}=\frac{(T-[\tau T])^{-2} \sum_{t=[\tau T]+1}^{T}\left(\sum_{i=[\tau T]+1}^{t} \tilde{v}_{i \tau}\right)^{2}}{[\tau T]^{-2} \sum_{t=1}^{[\tau T]}\left(\sum_{i=1}^{t} \hat{v}_{i \tau}\right)^{2}}
$$

where $\hat{v}_{i \tau}$ is the residual from the OLS regression of $x_{t}$ on $d_{t}$ for $t=1, \ldots,[\tau T]$ and $\tilde{v}_{i \tau}$ is the OLS residual from the regression of $x_{t}$ on $d_{t}$ for $t=[\tau T]+1, \ldots, T$.

Since the true change point, $\tau^{*}$, is assumed unknown Kim (2000), Kim et al. (2002) and Busetti and Taylor (2004) consider three statistics based on the sequence of statistics $\left\{K_{[\tau T]}, \tau \in \Lambda\right\}$, where $\Lambda=\left[\tau_{l}, \tau_{u}\right]$ is a compact subset of $[0,1]$, i.e.,

$$
\begin{aligned}
M S & =T_{*}^{-1} \sum_{s=\left[\tau_{l}\right]}^{\left[\tau_{u}\right]} K_{[s T]} ; \\
M E & =\ln \left\{T_{*}^{-1} \sum_{s=\left[\tau_{l}\right]}^{\left[\tau_{u}\right]} \exp \left[\frac{1}{2} K_{[s T]}\right]\right\} \\
M X & =\max _{s \in\left\{\left[\tau_{l}\right], \ldots,\left[\tau_{u}\right]\right\}} K_{[s T]}
\end{aligned}
$$

where $T_{*}=\left[\tau_{u}\right]-\left[\tau_{l}\right]+1$, and $\tau_{l}$ and $\tau_{u}$ correspond to the (arbitrary) lower and upper values assumed for $\tau^{*}$. Limit results and critical values for the statistics in (B.1) - (B.3) can be found in Harvey et al. (2006).

REMARK B.1. The procedure in (B.1) corresponds to the mean score approach of Hansen (1991), (B.2) is the mean exponential approach of Andrews and Ploberger (1994) and finally (B.3) is the maximum Chow approach of Davies (1977); see also Andrews (1993).

In order to test $H_{0}$ against the $\mathrm{I}(1)-\mathrm{I}(0)\left(H_{10}\right)$ hypothesis, Busetti and Taylor (2004) suggest the sequence of reciprocals of $K_{t}, t=\left[\tau_{l} T\right], \ldots,\left[\tau_{u} T\right]$. They define $M S^{R}, M E^{R}$ and $M X^{R}$ as the respective analogues of $M S$, $M E$ and $M X$, with $K_{[\tau T]}$ replaced by $K_{[\tau T]}^{-1}$ throughout. Furthermore, to test against an unknown direction of change (that is either a change from $\mathrm{I}(0)$ to $\mathrm{I}(1)$ or vice versa), they also propose $M S^{M}=\max \left[M S, M S^{R}\right]$, $M E^{M}=\max \left[M E, M E^{R}\right]$, and $M X^{M}=\max \left[M X, M X^{R}\right]$. Thus, tests which 
reject for large values of $M S, M E$, and $M X$ can be used to detect $H_{01}$, tests which reject for large values of $M S^{R}, M E^{R}$ and $M X^{R}$ can be used to detect $H_{10}$, and $M S^{M}, M E^{M}$, and $M X^{M}$ can be used to detect either $H_{01}$ or $H_{10}$.

Harvey et al. (2006) also introduce a set of modified test statistics such that the cdfs of the statistics under the null $\left(\mathrm{H}_{0}\right)$ and alternative $\left(\mathrm{H}_{1}\right)$ coincide asymptotically at an asymptotic critical value associated with a given significance level.

The first modified tests proposed where $M S_{m}=\exp \left(-b_{1} J_{1 T}\right) M S, M E_{m}=$ $\exp \left(-b_{2} J_{1 T}\right) M E$ and $M X_{m}=\exp \left(-b_{3} J_{1 T}\right) M X$, where $b_{k}, k=1,2,3$ are fixed constants and the modification also makes use of the unit root test proposed by Park (1990), defined as $J_{1, T}$ which consists of $T^{-1}$ times the Wald statistic for testing the joint hypothesis $\gamma_{k+1}=\ldots=\gamma_{9}=0$ in the regression,

$$
x_{t}=z_{t}^{\prime} \beta+\sum_{i=k+1}^{9} \gamma_{i} t^{i}+\text { error }, \quad t=1, \ldots, T .
$$

Note that under $\mathrm{H}_{0}, J_{1, T}$ is $O_{p}\left(T^{-1}\right)$ so that $\exp \left(-b_{k} J_{1 T}\right) \rightarrow 1, k=1,2,3$, and therefore $M S_{m}, M E_{m}$ and $M X_{m}$ are simply equivalent to the $M S, M E$ and $M X$ statistics.

The choice of $b_{k}, k=1,2,3$ ensures that, for a significance level, $100 a \%$, the corresponding asymptotic upper-tail critical value of $M S_{m}, M E_{m}$ and $M X_{m}$ under either $H_{0}$ or $H_{1}$ is identical to the corresponding upper-tail critical values of $M S, M E$ and $M X$ under $H_{0}$. These statistics have the same limiting distribution under $H_{0}$.

A further variante of modified procedures proposed by Harvey et al. (2006) is obtained by replacing $J_{1, T}$ with $J_{\min }=\min _{\tau \in \Lambda} J_{1,[\tau T]}$, where $J_{1,[\tau T]}$ is $T^{-1}$ times the Wald statistic for testing the joint hypothesis $\gamma_{k+1}=\ldots=\gamma_{9}=0$ in the regression,

$$
x_{t}=z_{t}^{\prime} \beta+\sum_{i=k+1}^{9} \gamma_{i} t^{i}+\text { error }, \quad t=1, \ldots,[\tau T] .
$$

Note that also in his case, under $\mathrm{H}_{0}, J_{\min }$ is $O_{p}\left(T^{-1}\right)$ so that $\exp \left(-b_{k}^{*} J_{\min }\right) \rightarrow$ $1, k=1,2,3$. Therefore, $M S_{m \min }=\exp \left(-b_{1}^{*} J_{\min }\right) M S, M E_{m \min }=$ $\exp \left(-b_{2}^{*} J_{\min }\right) M E$ and $M X_{m \min }=\exp \left(-b_{3}^{*} J_{\min }\right) M X$.

The reciprocal versions of these test, $M S_{m}^{R}, M E_{m}^{R}, M X_{m}^{R}$ and $M S_{m \min }^{R}, M E_{m \min }^{R}, M X_{m \text { min }}^{R}$, are constructed in a similar way, i.e., 
$M S_{m}^{R}=\exp \left(-b_{1} J_{1 T}\right) M S^{R}, M E_{m}^{R}=\exp \left(-b_{2} J_{1 T}\right) M E^{R}$ and $M X_{m}^{R}=$ $\exp \left(-b_{3} J_{1 T}\right) M X^{R}$; as well as $M S_{m \text { min }}^{R}=\exp \left(-b_{1}^{*} J_{\min }^{R}\right) M S^{R}, M E_{m \text { min }}^{R}=$ $\exp \left(-b_{2}^{*} J_{\min }^{R}\right) M E^{R}$ and $M X_{m \text { min }}^{R}=\exp \left(-b_{3}^{*} J_{\min }^{R}\right) M X^{R}$, where $J_{\min }^{R}=$ $\min _{\tau \in \Lambda} J_{[\tau T], T}$ and $J_{[\tau T], T}$ is $T^{-1}$ times the Wald statistic for testing the joint hypothesis $\gamma_{k+1}=\ldots=\gamma_{9}=0$ in the regression,

$$
x_{t}=z_{t}^{\prime} \beta+\sum_{i=k+1}^{9} \gamma_{i} t^{i}+\text { error }, \quad t=[\tau T]+1, \ldots, T .
$$

Finally, the modified tests against an unknown direction of change are simply given as, $M S_{m}^{M}=\exp \left(-b_{1} J_{1 T}\right) M S^{M}, M E_{m}^{M}=\exp \left(-b_{2} J_{1 T}\right) M E^{M}$, and $M X_{m}^{M}=\exp \left(-b_{3} J_{1 T}\right) M X^{M}$; as well as $M S_{m \min }^{M}=\exp \left(-b_{1}^{*} \min \left[J_{\min }, J_{\min }^{R}\right]\right) M S^{M}$, $M E_{m \min }^{M}=\exp \left(-b_{2}^{*} \min \left[J_{\min }, J_{\min }^{R}\right]\right) M E^{M}$ and $M X_{m \min }^{M}=\exp \left(-b_{3}^{*} \min \left[J_{\min }, J_{\min }^{R}\right]\right) M X^{M}$.

\section{B.3. Test outcomes}

Table B.1 gives the test outcomes for the null of constant persistence of the predictors considered in Section 5. We decided upon visual inspection whether a constant or a constant with linear trend is to be modeled as deterministic component $d_{t}$. Except for E12, there is serious evidence of timevarying persistence of the examined series. 


\begin{tabular}{|c|c|c|c|c|c|c|c|c|c|c|c|c|c|c|c|c|}
\hline & $\mathrm{D} 12^{\tau}$ & ${\mathrm{E} 12^{\tau}}^{\top}$ & $\mathrm{dp}^{\top}$ & $\mathrm{dy}^{\tau}$ & $\mathrm{ep}^{\top}$ & $\mathrm{de}^{\mu}$ & $\mathrm{b} / \mathrm{m}^{\tau}$ & $\mathrm{tbl}^{\mu}$ & lty $^{\mu}$ & $\mathrm{tms}^{\top}$ & $\mathrm{dfy}^{\tau}$ & $\mathrm{dfr}^{\tau}$ & $\mathrm{ntis}^{\mu}$ & $\inf ^{\mu}$ & $\operatorname{ltr}^{\mu}$ & svar $^{\mu}$ \\
\hline MS & $9.119^{*}$ & 2.491 & $3.813^{*}$ & $3.768^{*}$ & $6.713^{*}$ & $5.863^{*}$ & $7.436^{*}$ & $6.098^{*}$ & 4.313 & $3.179^{*}$ & $7.003^{*}$ & $3.606^{*}$ & $10.967^{*}$ & $5.137^{*}$ & 2.720 & $41.441^{*}$ \\
\hline ME & $36.044^{*}$ & 1.595 & $16.517^{*}$ & $16.212^{*}$ & $98.137^{*}$ & $8.523^{*}$ & $24.970^{*}$ & $17.216^{*}$ & $6.814^{*}$ & $5.126^{*}$ & $25.232^{*}$ & $9.818^{*}$ & $64.374^{*}$ & $12.256^{*}$ & 3.035 & $158.885^{*}$ \\
\hline MX & $80.592 *$ & 7.037 & $44.904^{*}$ & $44.289^{*}$ & $208.545^{*}$ & $22.654^{*}$ & $62.046^{*}$ & $41.968^{*}$ & $19.183^{*}$ & $17.032^{*}$ & $59.253^{*}$ & $28.275^{*}$ & $139.791 *$ & $32.410^{*}$ & 14.903 & $329.544^{*}$ \\
\hline $\mathrm{MS}^{R}$ & 2.635 & 0.563 & 2.764 & $2.874^{*}$ & 2.033 & 0.791 & $39.887 *$ & 2.095 & $4.984^{*}$ & 0.781 & 1.699 & 1.513 & 0.515 & $21.232^{*}$ & $5.099^{*}$ & 0.282 \\
\hline $\mathrm{ME}^{R}$ & $2.180^{*}$ & 0.299 & $9.612^{*}$ & $11.079^{*}$ & 1.476 & 0.431 & $200.415^{*}$ & 2.171 & $9.380^{*}$ & 0.455 & 1.731 & 0.885 & 0.303 & $38.791^{*}$ & $8.701^{*}$ & 0.156 \\
\hline $\mathrm{MX}^{R}$ & 7.520 & 1.440 & $29.262^{*}$ & $32.893^{*}$ & 6.062 & 1.613 & $413.101 *$ & 11.815 & $27.018^{*}$ & 2.885 & 7.135 & 4.809 & 2.168 & $86.771^{*}$ & $25.546^{*}$ & 1.037 \\
\hline $\mathrm{MS}^{M}$ & $9.119^{*}$ & 2.491 & $3.813^{*}$ & $3.768^{*}$ & $6.713^{*}$ & 5.863 & $39.887^{*}$ & $6.098^{*}$ & 4.984 & 3.179 & $7.003^{*}$ & $3.606^{*}$ & $10.967 *$ & $21.232^{*}$ & 5.099 & $41.441^{*}$ \\
\hline $\mathrm{ME}^{M}$ & $36.044^{*}$ & 1.595 & $16.517^{*}$ & $16.212^{*}$ & $98.137 *$ & $8.523^{*}$ & $200.415^{*}$ & $17.216^{*}$ & $9.380^{*}$ & $5.126^{*}$ & $25.232 *$ & $9.818^{*}$ & $64.374^{*}$ & $38.791 *$ & $8.701^{*}$ & $158.885^{*}$ \\
\hline $\mathrm{MX}^{M}$ & $80.592 *$ & 7.037 & $44.904 *$ & $44.289 *$ & $208.545^{*}$ & 22.654 & $413.101^{*}$ & $41.968^{*}$ & $27.018^{*}$ & $17.032 *$ & $59.253^{*}$ & $28.275^{*}$ & $139.791^{*}$ & $86.771^{*}$ & $25.546^{*}$ & $329.544^{*}$ \\
\hline & & & $\begin{array}{r}0.399 \\
0.399\end{array}$ & & 200.040 & & & & 0.267 & & & & & & & \\
\hline & & & 0.145 & 0.1 & 1 & $7.393^{*}$ & & $4.7 \mathrm{~s}$ & 0.0 & & $9.026^{*}$ & & & & & \\
\hline $\mathrm{MX}_{m}$ & $22.079^{*}$ & $\begin{array}{l}6.084 \\
6.08\end{array}$ & $\begin{array}{l}1.210 \\
1.210\end{array}$ & $\begin{array}{l}1.112 \\
1.12\end{array}$ & $60.420^{*}$ & $20.224^{*}$ & 0.377 & 15.125 & 0.506 & $15.549^{*}$ & $27.026^{*}$ & $24.790^{*}$ & $96.194 *$ & $27.536^{*}$ & 14.792 & $319.456^{*}$ \\
\hline $\begin{array}{l}\mathrm{AS}_{m}^{R} \\
\mathrm{M}^{2}\end{array}$ & 1.203 & 0.515 & 0.310 & 0.309 & 0.960 & 0.726 & 1.816 & 0.968 & 0.318 & 0.739 & 1.056 & 1.397 & 0.388 & $18.767^{*}$ & $5.071^{*}$ & 0.276 \\
\hline $\mathrm{ME}_{m}^{R}$ & 0.435 & 0.249 & 0.107 & 0.113 & 0.316 & 0.374 & 0.348 & 0.603 & 0.098 & 0.406 & 0.651 & 0.751 & 0.189 & $31.612^{*}$ & $8.620^{*}$ & 0.150 \\
\hline $\mathrm{MX}_{m}^{R}$ & 2.217 & 1.256 & 0.968 & 1.018 & 1.884 & 1.441 & 3.355 & 4.269 & $\begin{array}{l}0.719 \\
0.790\end{array}$ & $\begin{array}{l}2.400 \\
2.648\end{array}$ & $\begin{array}{l}3.031 \\
3.402\end{array}$ & 4.247 & $\begin{array}{l}1.493 \\
1.493\end{array}$ & $73.753^{*}$ & $25.357 *$ & $\begin{array}{l}0.100 \\
1.005\end{array}$ \\
\hline $\mathrm{MS}_{m}^{M}$ & $3.730^{*}$ & $\begin{array}{l}2.250 \\
2.253\end{array}$ & 0.315 & $\begin{array}{l}0.296 \\
0.296\end{array}$ & $\begin{array}{l}1.004 \\
2.854\end{array}$ & $\begin{array}{l}1.441 \\
5.308\end{array}$ & $\begin{array}{l}1.000 \\
1.177\end{array}$ & 2.491 & 0.205 & $\begin{array}{l}2.048 \\
2.985\end{array}$ & $\begin{array}{l}. .402 \\
4.073^{*}\end{array}$ & $\begin{array}{l}3.241 \\
3.293\end{array}$ & 7.4501* & $18.403^{*}$ & 5.066 & $40.326^{*}$ \\
\hline $\mathrm{ME}_{m}^{M}$ & $5.627^{*}$ & 1.294 & 0.093 & 0.082 & $16.599 *$ & 7.267 & 0.133 & 4.105 & 0.057 & $4.498^{*}$ & $8.183^{*}$ & $8.130 *$ & $38.079 *$ & $30.854^{*}$ & $8.611^{*}$ & $152.096^{*}$ \\
\hline $\mathrm{MX}_{m}^{M}$ & $19.458^{*}$ & $\begin{array}{l}5.998 \\
5.998\end{array}$ & 0.851 & 0.776 & $53.540^{*}$ & 19.850 & 1.527 & 12.787 & 0.391 & $15.411^{*}$ & $25.033 *$ & $24.474^{*}$ & $90.458^{*}$ & $71.772^{*}$ & $25.326 *$ & $317.826^{*}$ \\
\hline & & & & & & & & & & & & & & & & \\
\hline & 3.96 & & $5.953^{*}$ & $5.491^{*}$ & 53.74 & & 11.7 & & $\begin{array}{l}0.4 \\
0.2\end{array}$ & & $45^{*}$ & & $55.894 *$ & $29 *$ & & $510^{*}$ \\
\hline 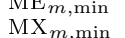 & $14.103^{*}$ & $\begin{array}{l}1.000 \\
4.869\end{array}$ & $20.055^{*}$ & $\begin{array}{l}5.489^{*} \\
18.832^{2}\end{array}$ & $129.624^{*}$ & $\begin{array}{l}15.597 \\
15.57\end{array}$ & $34.300^{*}$ & $29.823^{*}$ & $\begin{array}{l}0.092 \\
1.092\end{array}$ & $15.385^{*}$ & $40.212^{*}$ & $23.375^{*}$ & $124.152^{*}$ & $28.983^{*}$ & 14.851 & $325.401 *$ \\
\hline $\begin{array}{l}\mathrm{MS}_{m}^{R}, \min \\
\text { min }\end{array}$ & 1.423 & 0.531 & 1.623 & 1.737 & 1.531 & 0.754 & $29.706^{*}$ & 1.183 & 0.985 & 0.748 & 1.419 & 1.396 & 0.430 & $21.142^{*}$ & $5.082^{*}$ & $\begin{array}{c}0.279 \\
0.279\end{array}$ \\
\hline $\mathrm{ME}_{m, m i n}^{m, m i n}$ & 0.737 & 0.270 & $3.763^{*}$ & $4.565^{*}$ & 0.896 & 0.401 & $119.304^{*}$ & 0.935 & 0.862 & 0.421 & 1.260 & 0.768 & 0.232 & $38.550^{*}$ & $8.657^{*}$ & 0.153 \\
\hline $\mathrm{MX}_{m, \min }^{R, m}$ & 3.219 & 1.330 & $14.050^{*}$ & $16.439 *$ & 4.101 & 1.519 & $275.314^{*}$ & 5.821 & 3.632 & 2.717 & 5.565 & 4.305 & $\begin{array}{l}1.732 \\
1.732\end{array}$ & $86.316^{*}$ & $25.436 *$ & $\begin{array}{l}.1 .020 \\
1.020\end{array}$ \\
\hline $\mathrm{MS}_{m, \min }^{M,}$ & $3.895^{*}$ & 2.299 & 1.827 & 1.880 & $4.537 *$ & 5.475 & $26.554^{*}$ & 4.109 & 0.514 & 2.993 & $5.459 *$ & 3.227 & $9.562^{*}$ & $21.106^{*}$ & 5.079 & $40.840^{*}$ \\
\hline $\begin{array}{l}m, m \text { min } \\
\mathrm{ME}_{m}^{M} \min \end{array}$ & $8.220^{*}$ & 1.387 & $4.601^{*}$ & $4.843^{*}$ & $49.690 *$ & 7.736* & $98.843^{*}$ & $9.855^{*}$ & 0.378 & $4.616^{*}$ & $16.369 *$ & $8.096^{*}$ & $53.036^{*}$ & $38.467 *$ & $8.652^{*}$ & $155.636^{*}$ \\
\hline $\begin{array}{l}\operatorname{mX}_{m}^{M} \text { min } \\
\text { min }\end{array}$ & $24.490^{*}$ & $\begin{array}{l}6.290 \\
6.290\end{array}$ & $16.033^{*}$ & $16.729 *$ & $120.513^{*}$ & 20.824 & $233.713^{*}$ & 25.845 & 1.660 & $15.654^{*}$ & $41.810^{*}$ & $24.204^{*}$ & $118.130^{*}$ & $86.140^{*}$ & $25.420 *$ & $323.680^{*}$ \\
\hline
\end{tabular}

Table B.1. Persistence Change Test Results

Notes: * denotes significance at the $5 \%$ significance level. Critical values used taken from Harvey et al., 2006, p.451) for $T=\infty$. The superscripts $\tau$ and ${ }^{\mu}$ used on the variables analysed, indicates that the test results are obtained considering either detrended or demeaned data, respectively. 


\section{WORKING PAPERS}

\section{3}

01|13 Macroeconomic forecasting using lowfrequency filters

João Valle e Azevedo | Ana Pereira

02|13 Everything you always wanted to know about sex discrimination

Ana Rute Cardoso | Paulo Guimarães | Pedro Portugal

03|13 Is there a role for domestic demand pressure on export performance?

Paulo Soares Esteves | António Rua

04|13 Ageing and fiscal sustainability in a small euro area economy

Gabriela Castro | José R. Maria | Ricardo Mourinho Félix | Cláudia Rodrigues Braz

05|13 Mind the gap! The relative wages of immigrants in the Portuguese labour market Sónia Cabral | Cláudia Duarte

06|13 Foreign direct investment and institutional reform: Evidence and an application to Portugal

Paulo Júlio | Ricardo Pinheiro-Alves | José Tavares

07|13 Monetary policy shocks: We got news! Sandra Gomes | Nikolay Iskrev | Caterina Mendicino

08|13 Competition in the Portuguese Economy: Estimated price-cost margins under imperfect labour markets

João Amador | Ana Cristina Soares

09|13 The sources of wage variation: a threeway high-dimensional fixed effects regression model

Sonia Torres | Pedro Portugal | John T. Addison | Paulo Guimarães
10|13 The output effects of (non-separable) government consumption at the zero lower bound

Valerio Ercolani | João Valle e Azevedo

11|13 Fiscal multipliers in a small euro area economy: How big can they get in crisis times?

Gabriela Castro | Ricardo M. Felix | Paulo Julio | Jose R. Maria

12|13 Survey evidence on price and wage rigidities in Portugal

Fernando Martins

13|13 Characterizing economic growth paths based on new structural change tests Nuno Sobreira | Luis C. Nunes | Paulo M. M. Rodrigues

14|13 Catastrophic job destruction Anabela Carneiro | Pedro Portugal | José Varejão

$15 \mid 13$ Output effects of a measure of tax shocks based on changes in legislation for Portugal

Manuel Coutinho Pereira | Lara Wemans

16|13 Inside PESSOA - A detailed description of the model

Vanda Almeida | Gabriela Castro | Ricardo M. Félix | Paulo Júlio | José R. Maria

17|13 Macroprudential regulation and macroeconomic activity

Sudipto Karmakar

18|13 Bank capital and lending: An analysis of commercial banks in the United States Sudipto Karmakar | Junghwan Mok 


\section{4}

1|14 Autoregressive augmentation of MIDAS regressions

Cláudia Duarte

2|14 The risk-taking channel of monetary policy - exploring all avenues

Diana Bonfim | Carla Soares

3|14 Global value chains: Surveying drivers, measures and impacts

João Amador | Sónia Cabral

4|14 Has US household deleveraging ended? a model-based estimate of equilibrium debt

Bruno Albuquerque | Ursel Baumann | Georgi Krustev

5|14 The weather effect: estimating the effect of voter turnout on electoral outcomes in italy

Alessandro Sforza

6|14 Persistence in the banking industry: fractional integration and breaks in memory Uwe Hassler | Paulo M.M. Rodrigues | Antonio Rubia

7|14 Financial integration and the great leveraging Daniel Carvalho

8|14 Euro area structural reforms in times of a global crisis

Sandra Gomes

9|14 Labour demand research: towards a better match between better theory and better data

John T. Addison | Pedro Portugal | José Varejão
10|14 Capital inflows and euro area long-term interest rates

Daniel Carvalho | Michael Fidora

11|14 Misallocation and productivity in the lead up to the Eurozone crisis

Daniel A. Dias | Carlos Robalo Marquesz | Christine Richmond

12|14 Global value chains: a view from the euro area

João Amador | Rita Cappariello | Robert Stehrer

13|14 A dynamic quantitative macroeconomic model of bank runs

Elena Mattana | Ettore Panetti

14|14 Fiscal devaluation in the euro area: a model-based analysis

S. Gomes | P. Jacquinot | M. Pisani

15|14 Exports and domestic demand pressure: a dynamic panel data model for the euro area countries

Elena Bobeica | Paulo Soares Esteves | António Rua | Karsten Staehr

16|14 Real-time nowcasting the US output gap: singular spectrum analysis at work Miguel de Carvalho | António Rua 
1|15 Unpleasant debt dynamics: can fiscal consolidations raise debt ratios?

Gabriela Castro | Ricardo M. Félix | Paulo Júlio | José R. Maria

2|15 Macroeconomic forecasting starting from survey nowcasts

João Valle e Azevedo | Inês Gonçalves

3|15 Capital regulation in a macroeconomic model with three layers of default

Laurent Clerc | Alexis Derviz | Caterina Mendicino | Stephane Moyen | Kalin Nikolov | Livio Stracca | Javier Suarez | Alexandros P. Vardoulakis

4|15 Expectation-driven cycles: time-varying effects

Antonello D'Agostino | Caterina Mendicino

5|15 Seriously strengthening the tax-benefit link

Pedro Portugal | Pedro S. Raposo

6|15 Unions and collective bargaining in the wake of the great recession

John T. Addison | Pedro Portugal | Hugo Vilares

7|15 Covariate-augmented unit root tests with mixed-frequency data

Cláudia Duarte

8|15 Financial fragmentation shocks

Gabriela Castro | José R. Maria | Paulo úlio | Ricardo M. Félix

9|15 Central bank interventions, demand for collateral, and sovereign borrowing cost Luís Fonseca | Matteo Crosignani | Miguel Faria-e-Castro
10|15 Income smoothing mechanisms after labor market transitions

Nuno Alves | Carlos Martins

11|15 Decomposing the wage losses of displaced workers: the role of the reallocation of workers into firms and job titles Anabela Carneiro | Pedro Raposo | Pedro Portugal

12|15 Sources of the union wage gap: results from high-dimensional fixed effects regression models

John T. Addison | Pedro Portugal | Hugo Vilares

13|15 Assessing european firms' exports and productivity distributions: the compnet trade module Antoine Berthou | Emmanuel Dhyne | Matteo Bugamelli | Ana-Maria Cazacu | Calin-Vlad Demian | Peter Harasztosi | Tibor Lalinsky | Jaanika Meriküll | Filippo Oropallo | Ana Cristina Soares

14|15 A new regression-based tail index estimator: an application to exchange rates

João Nicolau | Paulo M. M. Rodrigues

15|15 The effect of bank shocks on firm-level and aggregate investment João Amador | Arne J. Nagengast

16|15 Networks of value added trade João Amador | Sónia Cabral

17|15 House prices: bubbles, exuberance or something else? Evidence from euro area countries Rita Fradique Lourenço | Paulo M. M. Rodrigues 


\section{6}

1|16 A mixed frequency approach to forecast private consumption with ATM/POS data Cláudia Duarte | Paulo M. M. Rodrigues | António Rua

2|16 Monetary developments and expansionary fiscal consolidations: evidence from the EMU António Afonso | Luís Martins

3|16 Output and unemployment, Portugal, 2008-2012

José R. Maria

4|16 Productivity and organization in portuguese firms

Lorenzo Caliendo | Luca David Opromolla | Giordano Mion | Esteban Rossi-Hansberg

5|16 Residual-augmented IVX predictive regression

Matei Demetrescu | Paulo M. M. Rodrigues 


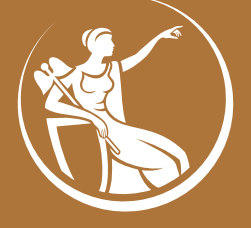

